\title{
Edge flame structure in a turbulent lifted flame: a direct numerical simulation study
}

\author{
Shahram Karami ${ }^{\mathrm{a}, *}$, Evatt R. Hawkes ${ }^{\mathrm{b}, \mathrm{a}}$, Mohsen Talei ${ }^{\mathrm{c}}$, Jacqueline H. Chen ${ }^{\mathrm{d}}$ \\ ${ }^{a}$ School of Photovoltaic and Renewable Energy Engineering, The University of New South Wales, Sydney, NSW 2052, Australia \\ ${ }^{b}$ School of Mechanical and Manufacturing Engineering, The University of New South Wales, Sydney, NSW 2052, Australia \\ ${ }^{c}$ School of Mechanical Engineering, University of Melbourne, Melbourne, 3010 Australia \\ ${ }^{d}$ Combustion Research Facility, Sandia National Laboratories, Livermore, California, CA 94551, United States of America
}

\begin{abstract}
This paper presents a statistical analysis of edge flames in a turbulent lifted flame using direct numerical simulation (DNS). To investigate the dynamics of edge flames, a theoretical framework describing the edge-flame propagation velocity as a function of propagation velocities of mixture-fraction and product-mass fraction iso-surfaces at the flame base is used. The correlations between these propagation velocities and several other variables are then studied, including iso-surface curvatures, iso-surface orientations, strain rates, scalar dissipation rate and gradients of product mass fraction. The contribution of these parameters to the overall behaviour of the edge flame is also investigated using conditional averaging on two-dimensional spatial locations at the flame base. The analysis reveals that the tangential and normal strain rates in addition to the curvatures and scalar dissipation rates have significant contributions to the overall behaviour of the edge flame. The elliptical motion of the flame base described in our earlier study [1] is extended to provide a clearer picture of how these various parameters affect the large fluctuations of edge-flame velocity observed at the flame base.
\end{abstract}

Keywords:

Lifted flame, Edge flame, DNS, Curvature, Strain rate, Scalar dissipation rate

\section{Introduction}

Lifted flames are present in many energy producing systems such as industrial burners, gas turbines and diesel engines. They can occur when a high velocity fuel jet is injected into a quiescent or a low velocity oxidising co-flow. If the jet velocity is high enough, the flame is not anchored at the nozzle lip but rather is stabilised some distance away from the nozzle. The location of the flame base has important implications for the design of the burners and the emissions they produce. As a result, a vast body of literature exists on understanding how lifted flames are stabilised [2-12]. Nevertheless, the stabilisation mechanism is still a poorly understood phenomenon. A number of theories such as the premixed flame theory [9], the edge-flame theory [13], the critical dissipation rate theory [14], and several theories involving a role played by large eddies $[12,15]$ have been proposed. As discussed in several review articles [15-17], aspects of these theories, and combinations of different theories, have varying levels of support when assessed against experimental data, and a final consensus on details of the stabilisation mechanism has not yet emerged.

\footnotetext{
${ }^{*}$ Corresponding author

Email address: s. karami@unsw.edu.au (Shahram Karami)
}

Among the theories that has the most support, however, according to Lyons' review [17], is the edge-flame theory. This concept originally proposed by Buckmaster [13], the leading edge of the flame is considered to be a partially premixed, selfpropagating edge flame that is centred at vicinity of the stoichiometric mixture fraction surface. Stabilisation is achieved as a result of the upstream self-propagation of the edge flame into flammable but as yet unburned mixtures, which balances the downstream flow. In the presence of low strain rates, the edge-flame structure has a tribrachial (or triple flame) structure consisting of two wings of premixed flames (one rich and one lean) and a tail of non-premixed (diffusion) flame. However, when the edge flame experiences high strain rates, one or both premixed branches collapse on the non-premixed tail creating a comet-shape structure.

There have been numerous studies of laminar edge flames, starting with the first experimental observation by Phillips [18], who showed that the edge flame is a self-propagating structure with a velocity balancing the oncoming flow. Kioni et al. [19] later observed the same structure in a similar experimental set-up. Kioni et al. [19] also used simulations to investigate the effects of strain rate on the structure of triple flames, showing that an increase in the strain rate resulted in the two premixed branches being merged on the diffusion tail. In another experimental study, Ko and Chung [20] measured 
the edge-flame propagation velocity in laminar non-premixed jet flames, reporting that it was much higher than the stoichiometric laminar burning velocity. It was also found that the propagation velocity was decreased when the fuel mass fraction gradient was increased. In an experimental study by Santoro et al. [21], the edge-flame structure was studied in a counter-flow mixing layer of methane and air. It was shown that flame extinction occurs when the edge flame experiences high strain rates, while a diffusion flame with no premixed wings was observed when the edge flame was subjected to sufficiently low strain rates. Away from these high and low strain limits, the flow and propagation velocities were balanced and a stable edge flame was observed.

Since the observation of the triple flame structure in experimental studies of laminar lifted flames, numerous analytical $[13,22,23]$ and numerical [8, 24-39] studies of this structure in different configurations were conducted. These studies are in general agreement about the influence of the strain rate on the edge-flame structure.

Analytical solutions can only be obtained under some restricted assumptions such as laminar flow, large activation energy and either neglecting heat release entirely or considering only weak heat release. For instance, Buckmaster and Weber [13] proposed a one-dimensional model for the edge-flame propagation in which the absolute speed can be negative, positive and zero. A negative absolute speed occurs when the strain rate is high leading to an extinction event whereas a positive absolute speed, i.e. ignition, coincides with low strain rate. When the flow velocity and edge propagation relative to the flow balance one another, the flame is stationary and the net speed is zero.

Ghosal and Vervisch [22] proposed an analytical expression for the edge-flame velocity in a symmetric laminar lifted flame assuming a large activation energy with a low, finite heat release rate. Their results showed that the edge-flame velocity departs from the laminar flame speed due to the effects of heat release and flame-front curvature. The importance of hydrodynamic effects (associated with the flame heat release rate) were also highlighted in several other analytical studies in the literature $[8,22,40]$. An interested reader is referred to an article by Buckmaster [23] provides a comprehensive review of analytical analyses of edge flames in different scenarios.

Detailed chemistry numerical simulations employing geometrically simple configurations, have also been used to study of laminar edge-flame characteristics [27, 34, 36]. The edgeflame structure in a scalar mixing layer between methanol and air was studied by Echekki and Chen [34]. Consistent with previous analytical and numerical studies $[8,40]$, Echekki and Chen found that the edge-flame propagation velocity depends on hydrodynamic effects. In a separate study by Im and Chen [27], a hydrogen-air triple flame was disturbed by inducing a pair of counter-rotating vortices. Im and Chen [27] observed that the edge-propagation velocity was strongly correlated by the flame stretch and the flame-front curvature, rather than the scalar dissipation rate.

The local structure of turbulent edge flames and flow dy- namics have been investigated using various laser-based measurements including particle image velocimetry (PIV), cinema particle imaging velocimetry (CPIV), laser-induced fluorescence (LIF), planar laser-induced fluorescence (PLIF), highspeed tomographic particle image velocimetry (TPIV), laserinduced predissociation fluorescence (LIPF), Rayleigh scattering and Raman-Rayleigh scattering [2-4, 6, 7, 11, 12, 4156]. Tribrachial structures have been observed in some of these measurements. For instance, Mansour [45] observed the rich wing and diffusion tail using LIPF measurements of $\mathrm{OH}$ radicals while Watson et al. [47] observed the rich and lean branches and the diffusion tail by adopting CH-PLIF measurements. The similarity of these visual observations of tribrachial structures to those observed in laminar triple flames suggests that edge flames, as self-propagating structures, play an important role in the stabilisation process [12, 46, 47, 57].

Understanding the stabilisation process and dynamics of edge flames requires measurements of the flow velocity and the relative edge-flame propagation velocity. Significant fluctuations of these velocities and lifted height have been observed in various experimental studies of lifted turbulent flames[12, 41, 45, 47]. Several experimental studies reported the instantaneous, two-dimensional flow velocity fields in the region of the flame base (without conditioning on instantaneous flame locations) $[3,42,46,47,52,57,58]$. It was observed that the streamlines of the flow diverged at the flame base due to the effects of heat release [3, 12, 45, 46, 52, 57]. A consequence of this flow divergence is a decrease in the flow velocity in the region of the flame base. This was proposed to allow the propagation of the edge flames into these low velocity regions. These observations are consistent with the earlier mentioned observations [18-21, 59] and theoretical results [13, 22, 23] for laminar triple flames. Some studies also present two-dimensional measurements of the flow velocity conditioned on the instantaneous flame locations [3, 46, 57], and compared them with the laminar flame speeds. For instance, Muñiz and Mungal [3] observed that the fluid velocity at the flame base is less than three times the laminar flame speed, $S_{L}$, while Upatnieks et al. [46, 57] reported the flow velocity to be similar to the laminar flame speed on average. Given that these measurements were conditional on the flame locations, and that the flow velocity and the edge-flame propagation velocity relative to flow should balance on-average, this suggests that the flames propagated at speeds the order of $S_{L}$, thus providing support to the edge-flame theory of stabilisation.

Attempts to measure the relative edge-flame velocity were also made [41, 43, 60, 61]. The two-dimensional absolute edge-flame velocity (flow plus relative edge-flame propagation) is accessible by comparing the flame-base location in two sequential measurements, and the relative edge-flame propagation velocity can then be obtained via a simultaneous measurement of flow velocity. Marking the flame location was critical to these experiments and some used the evaporation of liquid PIV seeding particles [6], while others used PLIF of a radical species, such as $\mathrm{OH}$ or $\mathrm{CH}[47,57,60,61]$. These 
measurements of relative propagation speeds showed that although the relative speed was generally in the order $S_{L}$ it was not a constant but fluctuated typically between about 0 and $3 S_{L}$, with sometimes negative or large positive speeds being observed. However, because these measurements were entirely two-dimensional, it was unclear whether these fluctuations were the result of actual variations in the propagation speed or rather the result of out-of-plane motion. More recently, attempts were made to partially alleviate this problem using measurements of the out-of-plane component of flow velocity. Boxx et al. [41] recently used a combination of a two-camera, stereoscopic PIV and an OH-PLIF imaging system with overlapping fields of view to measure the flow velocity including the out-of-plane component. Flame islands were observed upstream of the flame base and the appearance of these islands was found to be closely coupled to out-ofplane flow motion of the edge flames. In later work by Gordon et al. [60], measurements of the absolute flame displacement in two dimensions and all three components of velocity were reported. Compared with the previous studies which did not condition velocities on small out-of-plane component, a smaller variance of the relative propagation speed was noted; however, fluctuations of the relative speed were still present - for example the mean speed was different for edge-flames moving downstream to those moving upstream at some of the experimental conditions. Gordon et al. [60] also noted that the appearance of flame islands appeared to be the main mechanism of upstream motion of the flame, thus demonstrating the importance of out-of-plane flame motion. Further support that the three-dimensional structure is important was revealed in a recent study by Boxx et al. [41] who combined simultaneous OH-PLIF and line-of-sight chemiluminescence imaging to suggest that three-dimensional contortions of the flame structure could lead to large-magnitude changes the local orientation of flames, which suggests that flame propagation in out-of-plane directions can also be quite significant. Errors in all of the above measurements, e.g. in the measurement of the edge-flame locations and the relative edge-flame propagation velocities, also creates some doubt about the conclusions in these studies, e.g. see Hasselbrink and Mungal [6]. In summary, experimental methods have advanced from early PIV measurements of unconditional flow velocities, through measurements of flow velocities conditional on the instantaneous flame locations, to recent work which has measured relative velocities of edge flames in two-dimensions. Fully threedimensional measurements of relative flame velocities are still lacking.

As discussed earlier the significant fluctuations of the flow velocities conditioned at the flame base and lifted height are expected to be related to other parameters like scalar dissipation rates and strain rates. Therefore, in some experiments, attempts were made to measure these parameters. For instance, the scalar dissipation rate at the flame base was determined in experimental studies by Su et al. [12], Noda et al. [62] and Schefer et al. [11]. The measurements were two-dimensional, so only a two-dimensional representation of the scalar dissi- pation rate was available, although corrections were applied to estimate three-dimensional scalar dissipation rate. They all are in agreement that the scalar dissipation rate at the flame base is significantly lower than the critical stoichiometric extinction value from the counterflow diffusion flame. However, none of these works discuss correlations of the dissipation rate with velocity, despite the fact that a correlation is expected given previous work on laminar edge flames [8, 13, 24-26]. Two-dimensional measurements of strain rate $[4,60,63]$ and vorticity [60] in the vicinity of the flame base have been reported, and recently tomographic three-dimensional measurements of vorticity were also demonstrated [64], but attempts have not yet been made to determine how these quantities correlate with the relative edge-flame speed. As such, instantaneous and simultaneous measurements of all parameters of interest at the flame base, including scalar dissipation rate, curvatures of mixture-fraction and product-mass fraction iso-surfaces, strain rates and edge-flame relative propagation speeds are still out of reach for experiment.

An alternative approach is direct numerical simulation (DNS). DNS is a powerful tool that can provide a detailed understanding about the effect of important parameters on the edge-flame structure. For example, the characteristics of triple flames have been analysed in simple two-dimensional configurations [28, 30, 33, 37, 65, 66]. Mastorakos et al. [66] studied autoigntion in a two-dimensional mixing layer and observed that the mixture with the most reactive mixture fraction autoignited and subsequently propagated as an edge flame. Jiménez and Cuenot [28] studied the dynamics of an edge flame in presence of recirculating hot gases. It was shown that the edge flame propagates at a nearly constant speed, independent of the turbulence level, and is controlled by large-scale structures. In another study, a locally ignited edge flame in an inhomogeneous mixing layer in three-dimensional decaying turbulence was studied by Chakraborty and Mastorakos [30] using single-step chemistry. They observed that increasing the mixture-fraction gradients, induced by reducing the thickness of the mixing layer, slowed down the edge propagation represented by the displacement speed of the fuel mass-fraction iso-surface.

However, due to its high computational cost, DNS of edge flames in turbulent jets are limited to a few in the literature [39, 67-69]. For example, Pantano [39] used DNS with reduced chemistry to study non-premixed flame extinction in a methane-air jet flame. He developed a theoretical framework in which the edge-flame propagation was described as a function of the propagation velocities of the iso-surfaces of mixture fraction and a reacting scalar and the alignment of these iso-surfaces. His analysis of the resulting edgepropagation velocity around extinction holes showed that this velocity is largely controlled by the local rate of scalar dissipation. Hawkes et al. [67], in study of a turbulent temporally evolving nonpremixed plane-jet flame, observed that the edgeflame speed shows a strong negative correlation with scalar dissipation during extinction while a positive correlation was observed during reignition at low and intermediate scalar dis- 
sipation rates. Yoo et al. [68] studied a hydrogen lifted flame in a hot coflow condition, where the coflow temperature is sufficiently high to support autoignition.

The above-mentioned DNS studies have provided some useful information about the behaviour of edge flames in various turbulent flows (jet flame extinction, homogeneous turbulence ignition, etc.); however, none of these addressed a lifted flame in a non-autoignitive environment. This topic was recently tackled in another DNS study of the stabilisation mechanism in a turbulent lifted flame performed by the authors [1]. Therein it was shown that the flow on average balances the relative propagation of the edge flame, thus supporting the edgeflame stabilisation theory. However, significant fluctuations was observed in the lifted height, the propagation velocity and the flow velocity at the flame base. Conditioning of the net flame velocity on streamwise and transverse location revealed an elliptical clockwise motion of the edge flames around the average stabilisation point. It was proposed that this motion is connected with the passage of large eddies, in a manner mostly consistent with hypotheses put forward by $\mathrm{Su}$ et al. [12]. This was also consistent with the hypothesis put forth in an earlier DNS study of the near field of a turbulent lifted hydrogen/air jet flame in a heated coflow stabilised primarily by autoignition in which Yoo et al. [68] revealed the correlation of the passage of large-scale coherent flow structures and the relative position of the flame base, with the coherent motion inducing a cyclic motion of the flame base in the transverse and axial directions about a mean lift-off height.

In our previous DNS study of the stabilisation mechanism in a turbulent lifted flame [1], we also demonstrated that fluctuations of the local edge-flame propagation velocity were significant and led to significant fluctuations of lifted height, but did not investigate the cause of these fluctuations nor relate them to other important parameters. As explained earlier, experimental studies have also observed significant lifted height fluctuations [41, 46, 51, 70], and the very limited twodimensional estimates of edge-flame relative velocities that are available [26, 28, 30, 33, 37, 65] have likewise not been correlated with other parameters.

The aim of this paper is therefore to address this gap by providing a comprehensive analysis of the effects of important parameters on the edge-flame propagation velocity using DNS $[1,71]$. The investigated parameters include the displacement speeds of the product mass-fraction and mixture-fraction isosurfaces, the orientations of the normal vectors to these isosurfaces, their curvatures, strain rates, the dissipation rate of mixture fraction, and gradients of product mass fraction and mixture fraction. This article will also investigate the reasons for the observed large fluctuations of the edge-flame velocities as well as the observed on-average departures of the relative edge-flame propagation speed from the laminar burning velocity.

The paper is organised as follows: first, the numerical method and simulation parameters are briefly described. Next, a statistical analysis of the above-mentioned parameters will shed light on the edge-flame dynamics. Then, the picture pro- vided in the statistical analysis is synthesised. Finally, conclusions with suggestions for the future work are provided.

\section{Computational approach}

The DNS were completely described in Karami et al. [1], so only a brief description of key details is reported here for orientation. Figure 1 shows a schematic of the computational domain and the boundary conditions used to simulate the shown slot-jet lifted flame. Table 1 shows the list of parameters employed.

The DNS code S3D SC $[1,71,72]$ which is a modified version of the detailed chemistry code S3D [73] was used. The conservation equations of mass, momentum, sensible energy and fuel and oxidiser mass fraction with a single-step chemistry model were solved in a non-dimensional form. In this model, the fuel consumption rate is given by:

$$
\dot{\omega}_{F}=\frac{1}{r} \dot{\omega}_{O}=-\mathrm{Da} \rho^{2} Y_{F} Y_{O} \exp \left[-\frac{\beta\left(1-T^{\prime}\right)}{1-\alpha\left(1-T^{\prime}\right)}\right],
$$

$D a$ is a Damköhler number, $\rho$ is density, $Y_{F}$ and $Y_{O}$ are fuel and oxidiser mass fraction, beta is the Zel'dovich number. Also alpha and $T^{\prime}$ are given by:

$$
\begin{aligned}
T^{\prime} & =\frac{(\gamma-1) T-1}{\frac{\alpha}{(1-\alpha)}}, \text { and } \\
\alpha & =\frac{(\gamma-1) T_{a d}-1}{(\gamma-1) T_{a d}},
\end{aligned}
$$

where $T_{a d}$ is the adiabatic flame temperature and $\gamma$ is the ratio of specific heats.

The activation energy, represented nondimensionally by the Zel'dovich number $(\beta)$, was modified [74] such that the dependency laminar flame speed to equivalence ratio for methaneair flame can be reproduced in the simple chemistry model. In this model, the, Zel'dovich number is given by:

$$
\beta(\phi)=\left\{\begin{array}{ll}
\beta_{0}\left[1+8.25(\phi-0.64)^{2}\right] & \phi \leq 0.64 \\
\beta_{0} & 0.65<\phi<1.07 \\
\beta_{0}\left[1+1.443(\phi-1.07)^{2}\right] & \phi \geq 1.07
\end{array},\right.
$$

where $\beta_{0}$ is 5.0 and $\phi$ is the local equivalence ratio. A comprehensive justification of the choice of chemistry model is described in our previous article [1].

The mean inlet axial velocity and fuel mass fraction were specified using a tanh-based profile. The velocity fluctuations at the inlet were imposed using an auxiliary homogeneous isotropic turbulence field, produced based on a prescribed turbulent energy spectrum with a turbulence intensity of $5 \%$.

Like S3D [73], the solver S3D_SC uses high-order accurate, low dissipation numerical schemes and a 3D structured, Cartesian mesh. Spatial derivatives were computed using an $8^{\text {th }}$ order central differencing scheme and time integration was performed with a 6 -stage, $4^{\text {th }}$ order, explicit Runge-Kutta method. To suppress numerical fluctuations at high wave numbers, a $10^{\text {th }}$ order filter [75] was applied every 10 time steps. A non-reflecting boundary condition was imposed at 


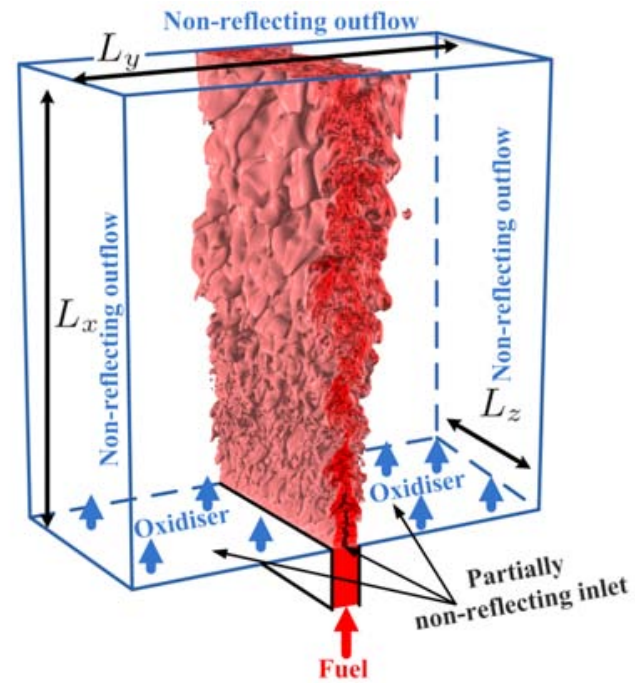

Figure 1: Schematic of the slot jet and specification of the boundary conditions.

the outflow while a partially non-reflecting boundary condition was imposed at the inflow. A periodic boundary condition was used in the spanwise direction.

To determine appropriate simulation parameters, the bulk parameters were approximately matched with an experimental jet-flame reported in [44]. Therein, $S_{L} / U_{j}=0.013$, $\delta_{L} / H=0.13$, and $\mathrm{Re}=5,280$ while in our simulation we have $S_{L} / U_{j}=0.016, \delta_{L} / H=0.16, \operatorname{Re}=5,280$.

The simulation was run for 18.0 jet flow through times, $t_{j}=$ $L_{x} / U_{j}$ (where the $L_{x}$ is the length of the computational domain in the streamwise direction), to obtain a statistically stationary, solution and the data from the last $12.0 t_{j}$ were used for the analysis. Further details of numerical methods are presented in Karami et al. [1].

\section{Mathematical background}

Now some necessary background regarding the identification of edge flames and their speeds relative to the flow will be developed in this section. Some of these materials are repeated from our earlier study [1] for the sake of completeness.

In premixed combustion, flame speeds are frequently obtained by calculating the displacement speed of a reacting scalar at a location in the flame that approximately tracks the locations of peak heat release [27, 34, 36, 37, 76-81].

In partially premixed flames the situation is more complicated. Observations from DNS [1, 39, 67, 82] show that the edge flames tend to remain anchored near to a particular mixture-fraction iso-surface. Therefore, following earlier studies of extinction and reignition $[39,67,82]$, and our previous study [1], we select an edge-flame marker that is the intersection of a mixture-fraction iso-surface and a product massfraction iso-surface. A slightly rich mixture fraction of 0.07 ,
Table 1: Numerical and physical parameters of the simulation

\begin{tabular}{lc}
\hline Jet width & $\mathrm{H}$ \\
Domain size $\left(L_{x} \times L_{y} \times L_{z}\right)$ & $16 H \times 24 H \times 8 H$ \\
Number of grid points $\left(N_{x} \times N_{y} \times N_{z}\right)$ & $800 \times 800 \times 400$ \\
Mean inlet jet Mach number $\left(U_{j e t}\right)$ & 0.48 \\
Laminar co-flow Mach number $\left(U_{c o-\text { flow }}\right)$ & 0.001 \\
Jet non-dimensional temperature & 2.5 \\
Co-flow non-dimensional temperature & 2.5 \\
Jet Reynolds number & 5,280 \\
Inlet velocity fluctuation & $5 \%$ \\
Fuel mixture fraction in fuel stream $\left(Y_{F, o}\right)$ & 1.0 \\
Oxidiser mixture fraction in oxidiser stream & 0.233 \\
(Yo,o $)$ & \\
Stoichiometric mixture fraction $\left(Y_{F s t}\right)$ & 0.055 \\
Stoichiometric oxidiser to fuel mass ratio $r$ & 4.0 \\
Heat release parameter $(\alpha)$ & 0.86 \\
Ratio of specific heat $(\gamma)$ & 1.4 \\
Prandtl number $(\operatorname{Pr})$ & 0.7 \\
Lewis number $($ Le=Sc/Pr) & 1.0
\end{tabular}

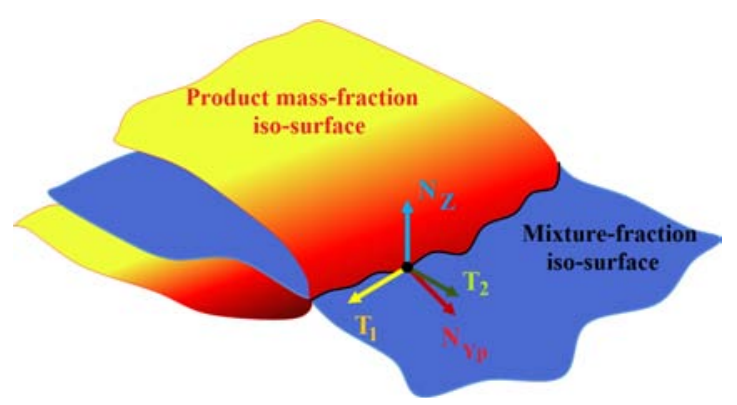

Figure 2: Various normal and tangential vectors at the flame base (the red/yellow surface is the product mass-fraction iso-surface, the blue surface is the mixture-fraction iso-surface and the solid black line is the edge flame).

corresponding to maximum laminar speed of a 1D flame, and a product mass fraction of 0.2 , corresponding to the location of the maximum reaction rate of a triple flame, were selected for these two iso-surfaces. Further details of the procedure to select these two iso-surfaces, the numerical schemes used to extract the intersection of iso-surfaces, and sensitivity analyses of the flame base locations to the selected mixture-fraction and product mass-fraction iso-surfaces can be found in Karami $e t$ al. [1].

To analyse the edge-flame motion, a coordinate system moving with the flow velocity is first defined - see figure 2 . In the figure: the normal to the mixture-fraction iso-surface is denoted $N_{Z}$ (pointing towards oxidiser); the normal to the product mass-fraction iso-surface is denoted $N_{Y_{p}}$ (pointing towards reactants); the tangent to the mixture-fraction isosurface which points along the flame edge is denoted $\boldsymbol{T}_{1}$; and finally, the tangent to the mixture-fraction iso-surface which is normal to $\boldsymbol{T}_{1}$, and pointing towards the reactants, is denoted 


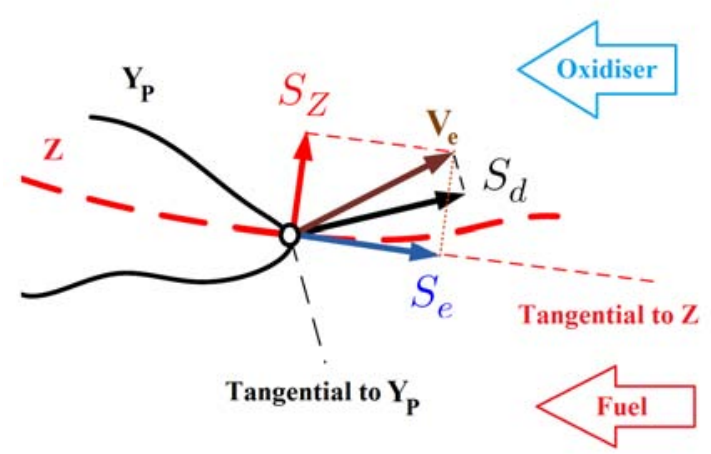

Figure 3: Schematic of edge-flame propagation along the mixturefraction iso-surface.

$\boldsymbol{T}_{2}$. It can be shown that these quantities are given by:

$$
\begin{aligned}
& \mathbf{N}_{Z}=-\frac{\nabla Z}{|\nabla Z|}, \mathbf{N}_{Y_{p}}=-\frac{\nabla Y_{p}}{\left|\nabla Y_{p}\right|}, \\
& \mathbf{T}_{1}=\frac{\mathbf{N}_{Y_{p}} \times \mathbf{N}_{Z}}{\left|\mathbf{N}_{Y_{p}} \times \mathbf{N}_{Z}\right|}, \\
& \mathbf{T}_{2}=\frac{\mathbf{N}_{Z} \times\left(\mathbf{N}_{Y_{p}} \times \mathbf{N}_{Z}\right)}{\left|\left(\mathbf{N}_{Y_{p}} \times \mathbf{N}_{Z}\right)\right|}, \text { and } \\
& \mathbf{T}_{3}=\frac{\mathbf{N}_{Y_{p}} \times\left(\mathbf{N}_{Y_{p}} \times \mathbf{N}_{Z}\right)}{\left|\left(\mathbf{N}_{Y_{p}} \times \mathbf{N}_{Z}\right)\right|} .
\end{aligned}
$$

Figure 3 shows a schematic, in the plane containing both $\mathbf{N}_{Z}$ and $\mathbf{N}_{Y_{p}}$, of the edge-point motion. Because the chosen coordinate system moves with the local flow velocity, only the motion of the iso-surfaces relative to the flow needs to be considered. Relative motion is a result of relative displacement of both the mixture-fraction and the product mass-fraction isosurfaces. The mixture-fraction iso-surface moves with the velocity of $S_{Z} \mathbf{N}_{Z}$, while the $Y_{P}=Y_{P}^{*}$ iso-surface moves with the velocity of $S_{d} \mathbf{N}_{Y_{p}}$, where the displacement speeds are given as $[34,83,84]$

$$
\begin{aligned}
& S_{Z}=\frac{1}{\rho|\nabla Z|}\left(\frac{\partial}{\partial x_{j}}\left(\frac{\mu}{\operatorname{Sc}} \frac{\partial Z}{\partial x_{j}}\right)\right), \text { and } \\
& S_{d}=\frac{1}{\rho\left|\nabla Y_{P}\right|}\left(\dot{\omega}_{P}+\frac{\partial}{\partial x_{j}}\left(\frac{\mu}{\operatorname{Sc}} \frac{\partial Y_{P}}{\partial x_{j}}\right)\right) .
\end{aligned}
$$

We denote the overall velocity of the edge point $\boldsymbol{V}_{e}$ and break this down in the orthonormal coordinates $\boldsymbol{N}_{Z}$ and $\boldsymbol{T}_{2}$ as:

$$
\mathbf{V}_{e}=S_{Z} \mathbf{N}_{Z}+S_{e} \mathbf{T}_{2},
$$

where $S_{e}=\boldsymbol{V}_{e} \bullet \boldsymbol{T}_{2}$ is the projection of $\boldsymbol{V}_{e}$ into the plane of the mixture-fraction iso-surface and needs to be determined. By taking the dot product of equation 6 with $N_{Y_{p}}$, it may be shown that

$$
S_{e}=\frac{S_{d}-k S_{Z}}{\sqrt{1-k^{2}}},
$$

where $k$ is the inner product of the normal vectors, $N_{Y_{p}} \bullet N_{Z}$.
Although the presentation here is slightly different, it can be verified that the final result for $S_{e}$ is the same edge speed used first by Pantano [39] to study extinction holes, and later by Hawkes et al. [67, 82] to study extinction and reignition, and also by Chakraborty et al. [29] to study ignitions.

To provide a better interpretation of the observed edgeflame speeds, some normalisation is required. In premixed combustion, where $S_{d}$ is the relevant speed, a densityweighted normalisation is often applied to correct for expansion across the flame as

$$
S_{d}^{*} / S_{L}=\frac{\rho S_{d}}{\rho_{u} S_{L}}
$$

where $\rho_{u}$ is the unburned gas density and $S_{L}$ is the laminar flame speed. This is equivalent to normalising by the $S_{d}$ value at the $Y_{p}=Y_{p}^{*}$ iso-surface in the unstrained 1D laminar flame, which we denote $S_{d, L}$. In the case of an edge flame, the normalisation is less straightforward because the expansion across the flame is less than $\rho_{u} / \rho$ due to streamline divergence around the edge flame. However, there does not seem to be a straightforward way to define an appropriate density correction because the amount of streamline divergence is dependent on the upstream mixture-fraction gradient. Therefore, following previous studies of edge flames [27, 29, 34, 36, 38, 67, 82], we simply adopt the same normalisation as used in premixed combustion to normalise all of the relevant speeds, i.e.:

$$
\frac{S_{e}^{*}}{S_{L}}=\frac{S_{e}}{S_{d, L}}, \text { and } \frac{S_{Z}^{*}}{S_{L}}=\frac{S_{Z}}{S_{d, L}} .
$$

To investigate the contribution of different terms in equations 5, the normalised displacement speeds are decomposed as [34]

$$
\begin{aligned}
& \frac{S_{d, n}^{*}}{S_{L}}=\frac{1}{S_{d, L}} \frac{N_{Y_{p}} \bullet \nabla\left(\rho D N_{Y_{p}} \bullet \nabla Y_{p}\right)}{\rho\left|\nabla Y_{p}\right|}, \\
& \frac{S_{d, t}^{*}}{S_{L}}=-\frac{1}{S_{d, L}} \frac{\mu}{\mathrm{Sc}} \frac{\kappa_{Y_{p}, m}}{\rho}, \\
& \frac{S_{d, r}^{*}}{S_{L}}=\frac{1}{S_{d, L}} \frac{\dot{\omega}_{P}}{\rho\left|\nabla Y_{p}\right|}, \\
& \frac{S_{Z, n}^{*}}{S_{L}}=\frac{1}{S_{d, L}} \frac{N_{Z} \bullet \nabla\left(\rho D N_{Z} \bullet \nabla Z\right)}{\rho|\nabla Z|}, \text { and } \\
& \frac{S_{Z, t}^{*}}{S_{L}}=-\frac{1}{S_{d, L}} \frac{\mu}{S c} \frac{\kappa_{Z, m}}{\rho},
\end{aligned}
$$

where $\kappa_{Y_{p}}$ is the curvature of the product mass-fraction iso-surface defined as $\nabla \bullet N_{Y_{p}}$ and $\kappa_{Z}$ is the mixture-fraction iso-surface curvature defined as $\nabla \bullet N_{Z}$. The subscript $n, t$ and $r$ represent the components resulting from diffusion normal to product mass-fraction iso-surfaces, diffusion tangential to product mass-fraction iso-surfaces, and reaction terms, respectively [34].

Strain can also be an important parameter for the edgeflame response [27, 34, 36, 38], and is often cast in terms of the total strain in the tangent plane of an iso-surface, or the component of strain in a particular directions (such as in the 


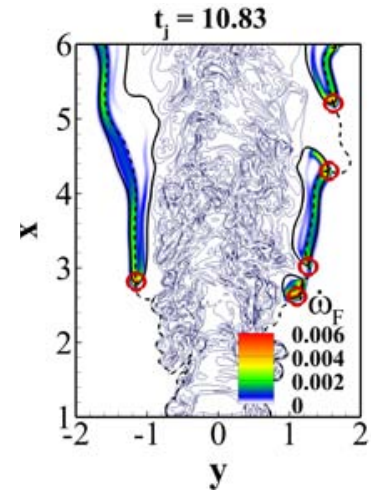

Figure 4: Contour plots of reaction rate and vorticity on the plane $z=0$. The solid black line is the product mass fraction, $Y_{p}$, of 0.2 and the dashed black line is the mixture fraction, $Z$, of 0.07 . The identified flame edges are marked as the centres of the red circles.

normal direction). The relevant strain rates discussed in this study are given as $[84,85]$

$$
\begin{aligned}
a_{t, Y_{p}} & =\left(\delta_{i j}-N_{Y_{p} i} N_{Y_{p} j}\right) \frac{\partial u_{i}}{\partial x_{j}}, \\
a_{n, Y_{p}} & =N_{Y_{p} i} N_{Y_{p} j} \frac{\partial u_{i}}{\partial x_{j}}, \\
a_{t, Z} & =\left(\delta_{i j}-N_{Z i} N_{Z j}\right) \frac{\partial u_{i}}{\partial x_{j}}, \\
a_{n, Z} & =N_{Z i} N_{Z j} \frac{\partial u_{i}}{\partial x_{j}}, \\
a_{T_{1}} & =T_{1 i} T_{1 j} \frac{\partial u_{i}}{\partial x_{j}} \text { and } \\
a_{T_{3}} & =T_{3 i} T_{3 j} \frac{\partial u_{i}}{\partial x_{j}} .
\end{aligned}
$$

In above, subscripts $t, Y_{p}$ and $t, Z$ represent the tangential strain rates on the respective iso-surfaces of those variables, $a_{n, Y p}, a_{n, Z}, a_{T_{1}}$, and $a_{T_{3}}$ represents the strain rate in the direction of the vectors $\boldsymbol{N}_{Y_{p}}, \boldsymbol{N}_{Z}, \boldsymbol{T}_{1}$, and $\boldsymbol{T}_{3}$ (Here $a_{n, Y p}$ and $a_{n, Z}$ correspond to normal strain rates for the $Y_{p}$ and $Z$ iso-surfaces.).

\section{Results and discussion}

\subsection{Orientation and motivating results}

For orientation, figure 4 shows an instantaneous contour plot of reaction rate and vorticity at the $z=0$ plane. The solid line shows the line of constant product mass fraction $Y_{p}=0.2$ and the dashed line represents the mixture fraction equal to 0.07 . The identified flame edges are marked as the centres of the red circles. It may be observed that the identified flame edges correspond well with regions of high reaction rate at the leading edge as well as at flame holes observed downstream.

The key results of our previous study [1] are now recounted. Figure 5 shows the PDF and mean total edge-flame velocity in

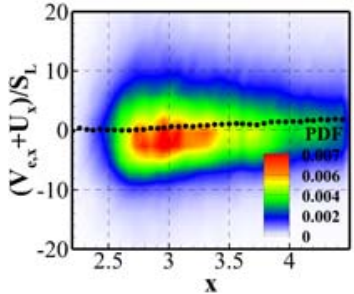

Figure 5: PDF and mean total edge-flame velocity in the streamwise direction $\left(V_{e, x}+U_{x}\right)$, conditioned on $\mathrm{x}$.
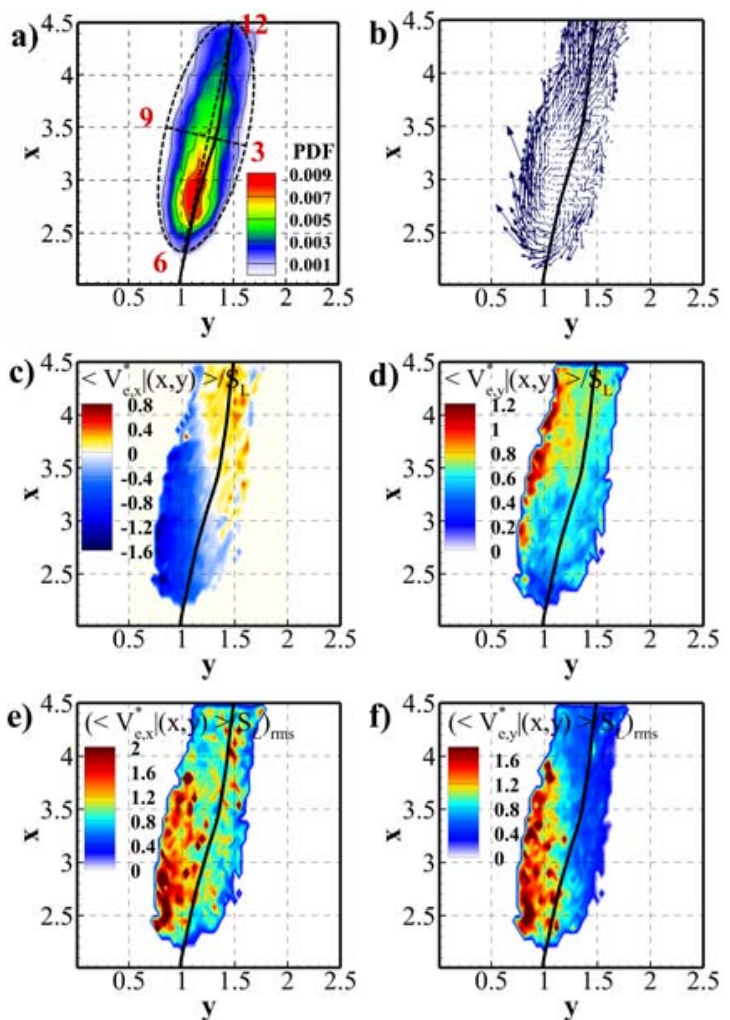

Figure 6: a) the joint PDF of the transverse and locations of edge flames. Overlaid is a representative clock face which will be referred to in order to facilitate discussions of the spatial structure in different regions. b)-f), the following quantities, conditionally averaged on the streamwise and transverse instantaneous location of edge flames: b) the vectors of relative velocity with the out-of-plane component added, c) streamwise edge propagation velocity, d) transverse edge propagation velocity e) the root-mean-square fluctuation of the streamwise edge propagation velocity and f) root-mean-square fluctuation of transverse edge propagation velocity. In all the figures, the solid line is the temporally and spatially Favre-averaged mixture fraction equal to 0.07 . 
the streamwise direction $\left(V_{e, x}+U_{x}\right)$, conditioned on $\mathrm{x}$. Although there are significant fluctuations, the conditional mean is very close to zero, i.e. the streamwise flow on average balances the upstream flame propagation, demonstrating that the flame is stabilised by edge-flame propagation.

Despite this on-average balance between streamwise flow and edge-flame propagation speed, fluctuations of the edgeflame locations are significant. To demonstrate this point, figure 6(a), reproduced from Ref. [1], presents the joint PDF of the streamwise and transverse locations of the flame edge. The solid line is the temporally and spatially Favre-averaged mixture fraction equal to 0.07 . As also shown in several experimental studies $[6,8,9,46,52,86]$, the flame base moves on the periphery of the jet with a higher possibility of the flame base being close to the mixture fraction of the maximum laminar flame speed, $Z_{m s}=0.07$, and significant fluctuations of the edge-flame locations occur. To explain the key reasons behind the fluctuations, figure 6(b), also reproduced from Ref. [1], shows vectors of the net edge-flame velocity (flow plus propagation). These vectors correspond to the net velocity at flame edges, observed by averaging over all $x, y$ planes and time, including the effect out-of-plane motion in each plane. The reader is directed to our earlier paper [1] for further details of the methodology used to construct this plot. The key point is that a clockwise rotating pattern is observed. In our earlier work, we suggested that this clockwise rotation is due to the passage of large eddies, along the lines of a previous suggestion by Su et al. [12], which refined earlier theories invoking a role of large eddies by Miake-Lye and Hammer [87], Kelman et al. [49], Tacke et al. [50] and Baillot \& Demare [88].

The previous work briefly noted that the mean relative displacement speeds were not always close to $S_{L}$, and that fluctuations of the edge-flame velocities were quite significant. The intent of the present article is to investigate the cause of these on-average departures of the relative edge-flame propagation speed from the laminar burning velocity, as well as to understand the causes of the speed fluctuations.

To orient later discussions relating to the clockwise motion of edge flames and to different regions within the spatiallyaveraged flame structure, a representative elliptical clock face is sketched in figure 6(a). We will also refer to the lean and rich sides, which should be understood as the on-average lean or rich sides, since the edge flames are always located instantaneously on the same mixture-fraction iso-surfaces.

Figures 6(c) and (d) show the normalised, densityweighted, streamwise and transverse components of the edge propagation velocity, conditionally averaged on the instantaneous streamwise and transverse locations of the flame base. The symbol $<\ldots \mid(x, y)>$ is the averaged quantity, doubly conditioned on streamwise and transverse locations of the edge flames. As may be observed, the streamwise flame propagation speed is mostly in the order of $S_{L}$ which is consistent with measurements [4, 12, 42-47], DNS in other configurations [36, 38, 39], and analytical models of edge-flame propagation [40]. The streamwise component of the edge-flame relative velocity is small but positive (i.e. the propagation is in the downstream direction) towards the upper right half of the plot (i.e. from around 10:30 o'clock through around 5 o'clock), while it is negative (i.e. upstream propagation) and larger than $S_{L}$ on the lower right hand side (from around 5 o'clock through 10:30). The transverse component is generally positive, i.e. the flame is tending towards outwards propagation, and significantly larger than $S_{L}$ in the upper left of the plot (from 9 o'clock through 12 o'clock), and towards very rich regions from 6 o'clock through 9 o'clock. The on-average cyclic edge-flame motion around the stabilisation point shown in figure 6 implies that the flame undergoes cyclic fluctuations of the relative speeds as well.

The root mean square (RMS) fluctuations of streamwise and transverse edge-propagation velocities are presented in figures 6(e) and (f), respectively, where the RMS fluctuation is defined for a quantity $q$ as:

$$
\langle q \mid(x, y)\rangle_{R M S}=\left(\left\langle q^{2} \mid(x, y)\right\rangle-\langle q \mid(x, y)\rangle^{2}\right)^{1 / 2} .
$$

As can be seen, the fluctuations of the streamwise propagation velocity are high, at least the order of the mean velocities, larger than $S_{L}$, and occur across all flame locations, but are somewhat stronger in the upstream and inner regions (bottom left of the plot). The fluctuations of the transverse flame propagation velocity are large on the rich side and relatively small on the lean side.

In summary the mean velocities can be significantly different from $S_{L}$ while the velocity fluctuations are also significant in comparison to the mean and to $S_{L}$. This motivates further investigation of the physical reasons underlying these trends which will be explained in forthcoming sections.

\subsection{Edge-velocity statistics}

In this section, the statistics of quantities obtained at all identified edge-flame locations are considered to learn which variables the speeds and orientations depend upon, in order to understand the overall dynamics. No spatial conditioning is applied in this section.

\subsubsection{Contributions of $S_{d}^{*}, S_{Z}^{*}$, and normal vector ori- entations}

The statistics of $S_{e}^{*}$, i.e. the edge-flame displacement speed along the mixture-fraction iso-surface towards reactants, are first investigated by looking into the correlations from its contributors, namely the term involving the displacement speed of product mass-fraction iso-surfaces, $S_{d}^{*} / S_{L} / \sqrt{1-k^{2}}$, and the term involving the displacement speed of the mixture-fraction iso-surfaces, $-k^{*} S_{Z} / S_{L} / \sqrt{1-k^{2}}$. The effect of the normal vector alignment, measured by $k=N_{Z} \bullet N_{Y_{p}}$, is also considered.

Figure 7(a) shows the joint PDF of $S_{e}^{*} / S_{L}$ and $S_{d}^{*} / S_{L} / \sqrt{1-k^{2}}$, whereas figure 7(b) shows the joint PDF of $S_{e}^{*} / S_{L}$ and $-k S_{z}^{*} / S_{L} / \sqrt{1-k^{2}}$. In both figures, the conditional mean of the ordinate on the abscissa is also shown as 

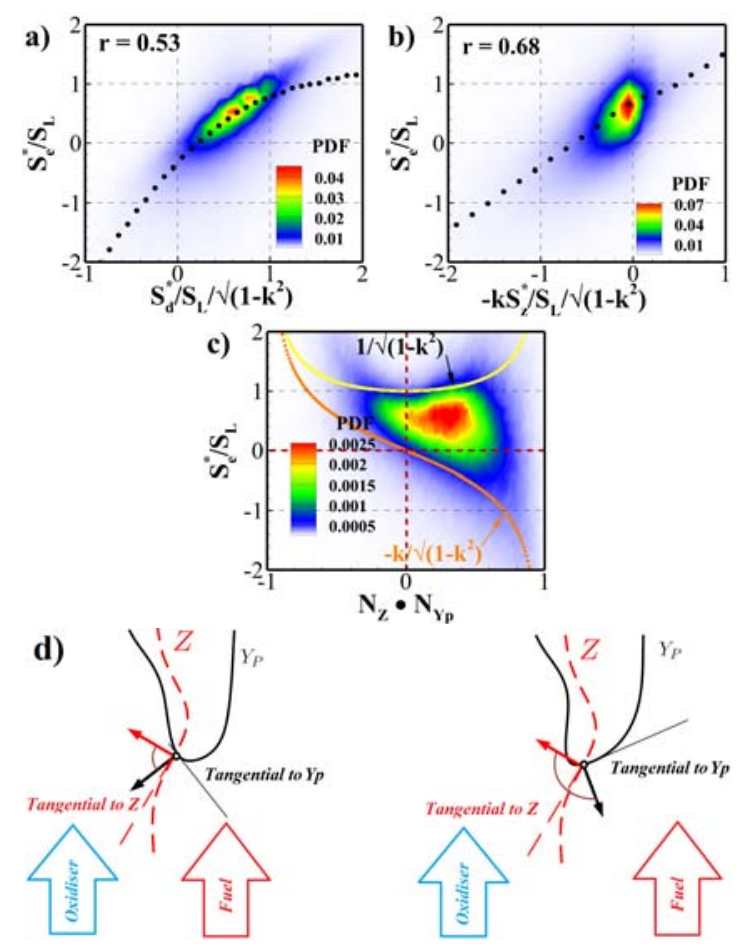

Figure 7: Contours of joint PDFs of $S_{e}^{*} / S_{L}$ with a) the contribution to $S_{e}^{*} / S_{L}$ of the normalised product mass-fraction displacement speed $\left(S_{d}^{*} / S_{L} / \sqrt{1-k^{2}}\right)$, b) the contribution to $S_{e}^{*} / S_{L}$ of the normalised mixture-fraction displacement speed $\left(-k S_{Z}^{*} / S_{L} / \sqrt{1-k^{2}}\right)$, c) the inner product $k$ of the normal vectors to product mass-fraction and mixture-fraction iso-surfaces, and (d) a schematic of two scenarios for the alignment of the normal vectors. a black line. Such conditional means will be shown throughout the article, but for brevity the fact that these lines represent the conditional means will not be mentioned henceforth. As shown in figure $7(\mathrm{a})$, the contribution of the product massfraction displacement speed, $S_{d}^{*} / S_{L} / \sqrt{1-k^{2}}$, is positive on average, although negative values are sometimes observed. The joint PDF is narrow and aligned with the conditional mean of $S_{e}^{*} / S_{L}$

In contrast, figure 7(b) shows that the term $-k S_{z}^{*} / S_{L} / \sqrt{1-k^{2}}$ is generally negative. The joint PDF shows a broader region around the conditional mean value compared with figure $7($ a). A positive correlation between $S_{e}^{*} / S_{L}$ and both terms is observed.

Figure 7(c) shows the joint PDF of $S_{e}^{*}$ and the inner product of the unit vectors normal to the mixture-fraction and product mass-fraction iso-surfaces, $k$. The yellow and orange dots show the terms $1 / S_{L} / \sqrt{1-k^{2}}$ and $-k / S_{L} / \sqrt{1-k^{2}}$ which appear as coefficients of $S_{d}^{*}$ and $S_{Z}^{*}$ in equation 7. It is observed that the joint PDF of $S_{e}^{*}$ and $k$ generally lies between the orange and yellow curves. The significance of the yellow curve is that it is the expected behaviour if $S_{d}^{*} / S_{L}$ is order of unity and $S_{Z}^{*} / S_{L} \approx 0$. This might be the case when a triple flame is stabilised in a mixing layer with very wide mixing thickness. The latter curve is the expected behaviour in the opposite situation $\left(S_{d}^{*} / S_{L} \approx 0\right.$ and $\left.S_{Z}^{*} / S_{L} \approx 1\right)$. Although this latter situation does not have physical significance (since there is no particular reason $S_{Z}^{*}$ should be order $S_{L}$ ), it does seem to bound the likely regions quite well, perhaps coincidentally.

The most likely value of $k=N_{Z} \bullet N_{Y_{p}}$ is positive, but significantly smaller than unity, i.e. the vectors are somewhat but not fully aligned. A near-unity value of $k$ would be expected in an unperturbed laminar flame, so non-unity values suggest effects of turbulent straining, which should tend to align (or antialign) both normal vectors with the most compressive strain direction. Concerning the direction of the alignment, two possible scenarios corresponding to the alignment of $\boldsymbol{N}_{Z}$ and $\boldsymbol{N}_{Y_{p}}$ are presented in figure 7(d), schematically. Figure 7(c) shows that a positive inner-product is more probable, i.e. the left schematic in figure 7(d). Further discussion of the reason for this bias requires an understanding of the spatially averaged picture, and this will be discussed later in the article.

\subsubsection{Responses to scalar dissipation rate}

It has been suggested that scalar dissipation contributes to the stabilisation mechanism of non-premixed flames [62, 89]. Therefore, in this section the effect of scalar dissipation rate on the edge-flame displacement speeds and orientations will be discussed.

Figure 8(a) shows the joint PDF of $S_{e}^{*} / S_{L}$ and logarithm of the normalised scalar dissipation rate and its conditional mean. The joint PDF shows a predominant negative correlation consistent with expectations from theory [8, 13, 22, 23, 90, 91] and the previous DNS study of flame holes by Pantano [39]. The most probable $S_{e}^{*}$ is approximately $0.6 S_{L}$. This value is close to the measurement of the flame propagation velocity in methane lifted flame by Upatnieks et al. [46], in which the 

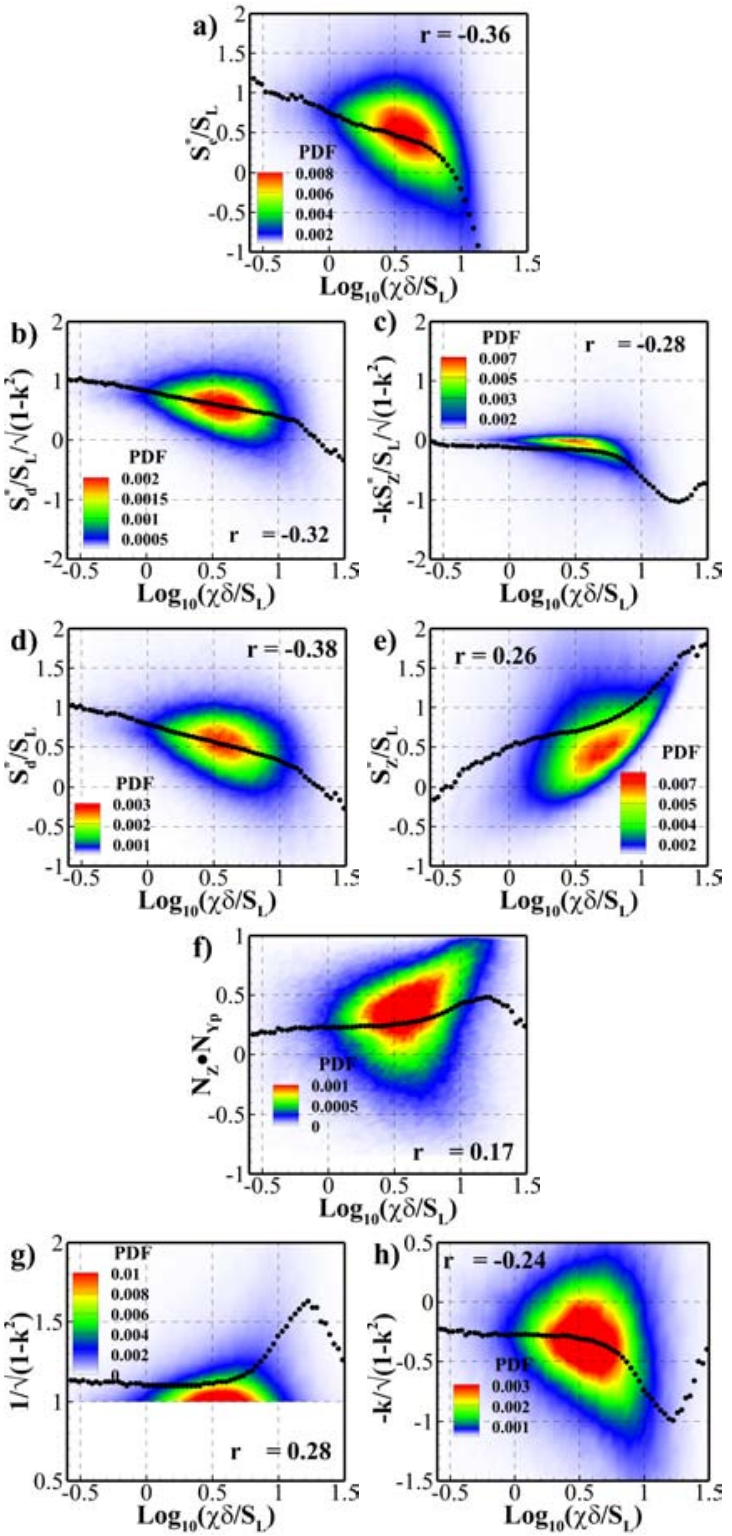

Figure 8: The joint PDFs with logarithm of the normalised scalar dissipation rate of the following quantities and their conditional means: a) $S_{e}^{*} / S_{L}$, b) the net contribution of normalised product mass-fraction displacement speed to $S_{e}^{*} / S_{L}$, c) the net contribution of normalised mixture-fraction displacement speed in $S_{e}^{*} / S_{L}$, d) $S_{d}^{*} / S_{L}$, e) $S_{Z}^{*} / S_{L}$, $\mathrm{f}$ ) the inner product of the normal vectors, $k, \mathrm{~g}$ ) the orientation term in contribution of $S_{d}^{*} / S_{L}$, and h) the orientation term in contribution of $S_{Z}^{*} / S_{L}$
Reynolds number of the jet and the ratio of jet velocity to laminar flame speed are similar to this study.

Figures 8(b) and (c) show the joint PDF of the contributions of the displacement speeds of the product mass-fraction (b) and mixture-fraction iso-surfaces (c) to $S_{e}^{*} / S_{L}$ with the logarithm of the normalised scalar dissipation rate, and their conditional means. Both quantities show a negative correlation with scalar dissipation rate.

Figure 8(b) shows a smooth transition from high to low values of $S_{d}^{*} / S_{L} / \sqrt{1-k^{2}}$ as scalar dissipation rate increases, with a somewhat sharper fall-off after $\chi S_{L} / \delta \approx 1$. However, the term $-k S_{Z}^{*} / S_{L} / \sqrt{1-k^{2}}$ (figure $8(\mathrm{c})$ ) which is negligible at low scalar dissipation rates, features a more sudden drop to negative value at high values of scalar dissipation rate, suggesting it plays the leading role in such conditions.

Figures 8(d) and (e) show the joint PDFs of $S_{d}^{*} / S_{L}$ (d) and $S_{Z}^{*} / S_{L}$ (e) with scalar dissipation rate. It is noted that both PDFs are broad. A negative correlation with scalar dissipation rate is exhibited by $S_{d}^{*} / S_{L}$, as expected, whereas $S_{Z}^{*} / S_{L}$ exhibits a positive correlation. The negative correlation of the propagation velocity of a reactive scalar with scalar dissipation rate has been observed in experimental [20] and numerical [40] studies of laminar triple flames. In an analytical study of counterflow premixed flames by Daou and Liñán [92], it was observed that by increasing the scalar dissipation rate, the propagation velocity decreases and the ignition front evolves to an extinction front, which is also consistent with the present results.

To explain the differences between the PDFs of the displacement speeds with and without the orientation factors included (e.g. that of $S_{d}^{*} / S_{L} \sqrt{1-k^{2}}$ in figure 8(b) versus that of $S_{d}^{*} / S_{L}$ in figure 8(d)), the joint PDF of the inner-product of the normal vectors, $k$, is shown in figure 8(f). A clear correlation is observed, with low levels of alignment more likely at lower dissipation rate, and higher levels of alignment more likely at higher dissipation rates. This correlation is consistent with local alignments of $\mathrm{OH}$ (a flame indicator) and mixture fraction gradients in study of turbulent temporally-evolving plane $\mathrm{CO} / \mathrm{H} 2$ jet flames by Hawkes et al. [93]. This trend of course affects the joint PDFs of the orientation coefficients for $S_{d}^{*} / S_{L}$ and $S_{Z}^{*} / S_{L}$ in equation 7 with scalar dissipation rate, which are shown in figures $8(\mathrm{~g})$ and $(\mathrm{h})$. As can be seen, the orientation coefficient of $S_{d}^{*}$ features a narrow PDF and a positive correlation with scalar dissipation rate, whereas the coefficient of $S_{Z}^{*} / S_{L}$ features a broad PDF and a negative correlation with scalar dissipation rate. For large dissipation rates, the effect of the orientation coefficients can be quite significant. The orientation coefficient for $S_{d}^{*} / S_{L}$ becomes large and positive while the orientation coefficient for $S_{Z}^{*} / S_{L}$ becomes large and negative. This is a result of the normal vectors becoming well aligned, i.e. $k$ increasing towards unity and thus the denominator becoming small. Such alignment is expected, because high dissipation rates are the result of persistent straining, which also tends to align scalar gradients, as noted in early studies of passive scalar mixing, for example study by Juneja and Pope [94]. 

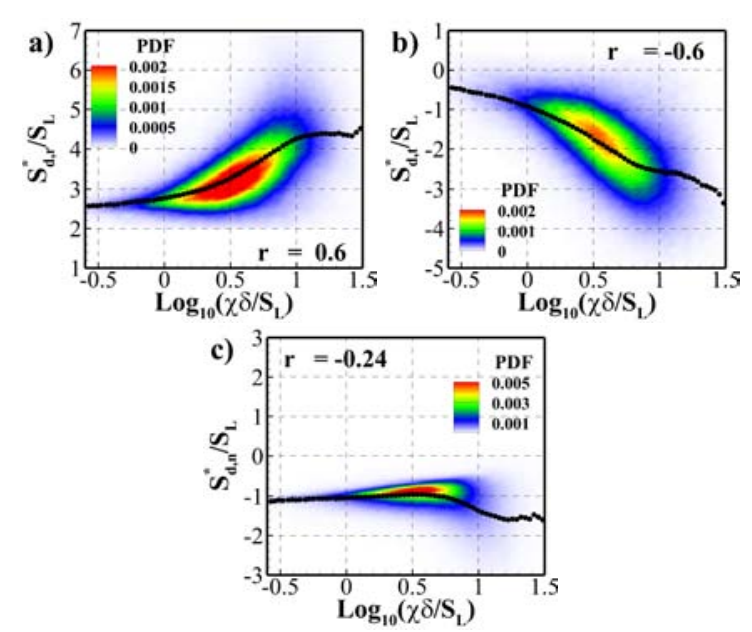

Figure 9: The joint PDFs of the components of the product massfraction propagation velocity a) $S_{d, r}^{*} / S_{L}$, b) $S_{d, t}^{*} / S_{L}$ and c) $S_{d, n}^{*} / S_{L}$ with the logarithm of normalised scalar dissipation rate.

Synthesising the picture from figures $8(\mathrm{a})-(\mathrm{g}), S_{d}^{*}$ plays the main role in the scalar dissipation rate response for moderate dissipation rates. The mixture-fraction iso-surface displacement speeds are not insignificant for moderate scalar dissipation rates but a small orientation factor reduces their overall role. However, at large dissipation rates, the normal vectors tend to become aligned, which steepens the response of the $S_{d}^{*}$-related term, and results in a large contribution from $S_{Z}^{*}$ to significantly increase the negative correlation with scalar dissipation rate, thus leading to more rapid extinction.

To further understand the response of $S_{d}^{*}$ to scalar dissipation, $S_{d}^{*}$ is broken down into components representing the effects of reaction, normal and tangential diffusion [34] - see equation 10.

The joint PDFs of the reaction, tangential and normal diffusion components of $S_{d}^{*}$ with scalar dissipation rate are presented in figure 9. It may be observed that the reaction term features a strong positive correlation with scalar dissipation rate whereas the tangential diffusion term exhibits a strong negative correlation. Figure 9(c) shows a weaker nonmonotonic contribution of the normal diffusion term, especially at moderate dissipation rates, and also a narrow PDF around the conditional mean. Comparison of figures 8(d) and 9 suggests that the positive correlation of the reaction term with scalar dissipation rate must be dominated by the negative correlation of the tangential diffusion term for moderate scalar dissipation rates, with the normal diffusion term playing a role at larger dissipation rates.

The reason behind these correlations is now investigated. We first note that because a one-step chemistry model is used with unity Lewis numbers, the thermochemical state-space is only two-dimensional if compressibility effects are ignored. Therefore, since the edge flames are located at fixed product mass-fraction and mixture-fraction iso-surfaces, the reaction rate itself only experiences small fluctuations due to com-
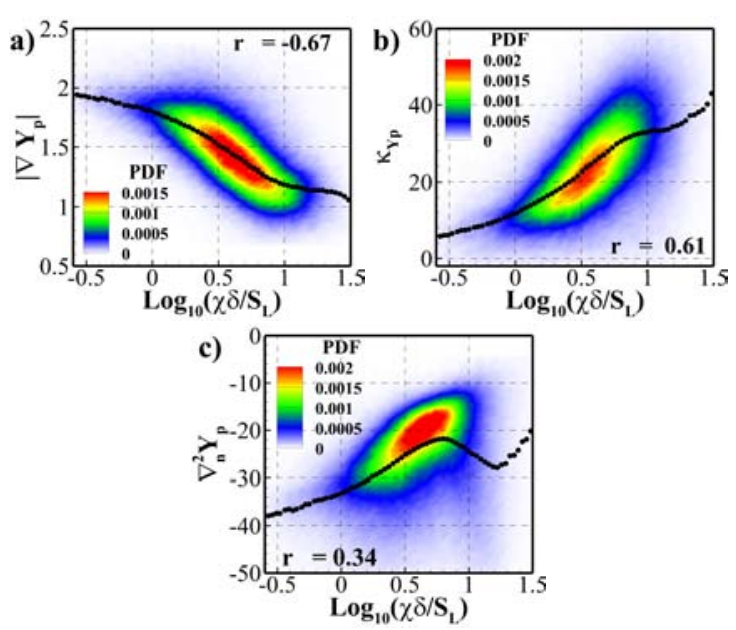

Figure 10: Contours of the joint PDFs with normalised scalar dissipation rate of: a) the magnitude of the product mass-fraction gradient, b) the product mass-fraction curvature, and c) the second derivative of the product mass fraction in the normal direction.

pressibility effects ${ }^{1}$. It can therefore be taken as a constant in this discussion. The reaction term is then simply proportional to the reciprocal of the magnitude of the product mass-fraction gradient, $1 /\left|\nabla Y_{P}\right|$, which scales with the thermal thickness. The normal diffusion term is approximately proportional to the second derivative of mass fraction in the normal direction $-\nabla_{n}^{2} Y_{P} /\left|\nabla Y_{P}\right|$ and the tangential term is curvature dependent. Figure 10(a) presents the joint PDF of $\left|\nabla Y_{P}\right|$ and scalar dissipation rate. In figure $10(\mathrm{a})$, a negative correlation of $\left|\nabla Y_{P}\right|$ and scalar dissipation rate may be observed. This can easily be understood in terms of the PDF of $k$, which shows that on average the normal vectors are not strongly aligned, and often close to perpendicular, implying that a compressive strain for mixture-fraction iso-surfaces, leading to high dissipation rates, corresponds more often to an extensive strain on product mass-fraction iso-surfaces. This correlation in turn leads to a positive correlation of the reaction term and scalar dissipation term (figure 9(a)).

As shown in equation 10, the tangential diffusion term is proportional to the curvature of the product mass-fraction isosurfaces. Figure 9(b) therefore suggests that there is an underlying correlation between the curvature and scalar dissipation rate. To confirm this, figure 10(b) shows the joint PDF of $\kappa_{Y p}$ and $\log _{10}\left(\chi \delta_{L} / S_{L}\right)$. A strong positive correlation is observed, as expected and reported in previous experimental and numerical studies of laminar lifted flames [59, 95]. Similarly to the above discussion, this may be explained considering the earlier result for the alignment of the normal vectors, shown in figure $7(\mathrm{c})$, which demonstrated that the most likely inner

\footnotetext{
${ }^{1}$ This is the case because in this one-step chemistry model with unity Lewis number, the thermochemistry is completely determined to within compressible fluctuations by mixture fraction and product mass fraction.
} 


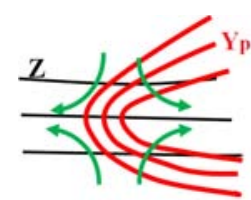

Low $\chi$

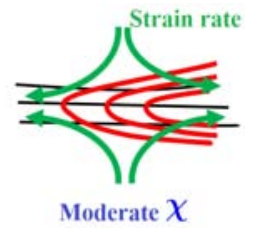

Moderate $\chi$
Figure 11: Indicative low- and moderate- scalar dissipation scenarios. The black lines depict mixture-fraction iso-surfaces, while the red lines represent product mass-fraction iso-surfaces. The green arrows show the strain direction.

product of the normal vectors was significantly smaller than unity. In this situation, compressive strain in the direction of the mixture-fraction normal, which leads to high dissipation rates, causes increases of the product mass-fraction curvature. Further discussion of curvature-related effects will be given in section 4.2.3.

To explain the trends for the normal diffusion component, figure 10(c) shows the joint PDF of $\nabla_{n}^{2} Y_{P}$ and $\chi$. As may be observed, $\nabla_{n}^{2} Y_{P}$ is negatively correlated in magnitude with scalar dissipation rate, but this is offset by a positive correlation between scalar dissipation rate and $1 /\left|\nabla Y_{P}\right|$, which can be inferred from figure 10(a). For moderate dissipation rates, the correlation with $\nabla_{n}^{2} Y_{P}$ is dominant, leading to a positive correlation for $S_{d, n}^{*} / S_{L}$ with scalar dissipation rate. In contrast, for larger dissipation rates $\left(\log _{10}\left(\chi \delta_{L} / S_{L}\right)>0.5\right)$, the correlation with $1 /\left|\nabla Y_{P}\right|$ dominates, leading to a negative correlation for $S_{d, n}^{*} / S_{L}$ with scalar dissipation rate.

In summary the above analysis of the trends for $S_{d}^{*}$ as surmised by figures 9 and 10 show that its decreasing dependence on scalar dissipation rate is due to a competition between tangential diffusion (decreasing $S_{d}^{*}$ with $\chi$ ) and an increasing reaction terms (increasing $S_{d}^{*}$ with $\chi$ ). These terms have opposite trends because compressive strain tends to cause the mixture-fraction gradient to align with it, which increases $\chi$, but decreases product mass-fraction gradients (increasing $S_{d, r}^{*}$ ) and increases product-mass fraction curvature (decreasing the $S_{d, t}^{*}$ component). To make this picture more clear, indicative low-strain and high-strain scenarios are sketched in figure 11

We now investigate the behaviour of the $S_{Z}^{*}$ components The joint PDFs of tangential and normal diffusion components of $S_{Z}^{*} / S_{L}$ with scalar dissipation rate are presented in figures 12(a) and (b). The normal diffusion term in figure 12(a) shows a positive correlation with scalar dissipation rate whereas a negative correlation is observed for the tangential diffusion term in figure 12(b). The normal diffusion term is dominant, explaining the positive correlation of $S_{Z}^{*}$ with scalar dissipation rate that was observed in figure $8(\mathrm{e})$.

The normal diffusion component is approximately proportional to $\nabla_{n}^{2} Z /|\nabla Z|$. Figure 12 (c) shows that $\nabla_{n}^{2} Z$ varies approximately quadratically with $|\nabla Z|$, which can be understood with the scalings $\nabla_{n}^{2} Z \approx 1 / \delta_{Z}^{2}$ and $|\nabla Z| \approx 1 / \delta_{Z}$, where $\delta_{Z}$ is a diffusive length scale. This explains the positive correlation observed between $S_{Z, n}^{*}$ and the scalar dissipation rate which
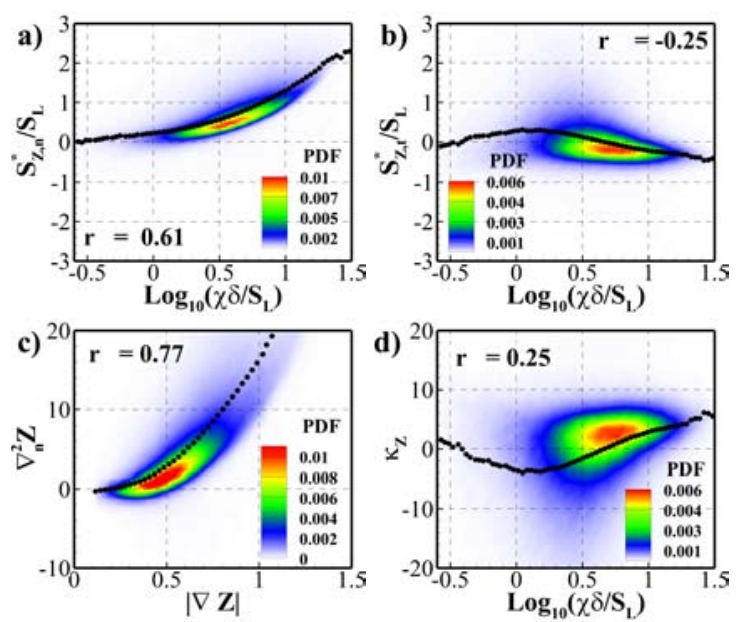

Figure 12: The joint PDFs of the components of the mixture-fraction propagation velocity a) $S_{Z, n}^{*}$, b) $S_{Z, t}^{*}$ with the logarithm of normalised scalar dissipation rate, c) Laplacian of mixture-fraction normal to mixture-fraction iso-surface with mixture-fraction gradient; and d) the joint PDF of the mixture-fraction curvature and the scalar dissipation rate.

scales as $1 / \delta_{Z}^{2}$ (figure $\left.12(a)\right)$.

To explain the relatively weaker positive correlation between $S_{Z, t}^{*}$ and scalar dissipation rate, figure 12(d) shows the joint PDF of $\kappa_{Z}$ and scalar dissipation rate. In the region that contains most of the sample, the correlation is positive. Extensive visual inspections of three-dimensional renderings of mixture-fraction iso-surfaces coloured by dissipation rate (not shown) suggest that this positive correlation results from specific eddy structures in the jet. The highest dissipation rates are observed to occur in structures which bulge out towards the oxidiser side, thus having a positive mixture-fraction curvature. (Further support for this is given later in the article.) As discussed above, higher gradients correspond to an even higher normal second derivative, which leads to the increase in the normal diffusion component of $S_{Z}^{*}$ with scalar dissipation rate.

\subsubsection{Responses to curvatures}

In fully premixed flames, it is well known that the curvature of reactive scalar iso-surfaces has a strong effect on local propagation speeds $[27,30,34,35,38,81,96-100]$, the effect being Lewis-number and equivalence-ratio dependent. Moreover, as discussed in previous literature, for near-unity Lewis-number conditions, edge-flame propagation speeds respond negatively to the curvature of product mass-fraction isosurfaces [20, 22, 27, 30, 81]. Although there is less discussion of their effect in the literature, in partially premixed flames the curvatures of mixture-fraction iso-surfaces are also potentially important.

The aim of this section is therefore to understand the effects of these two curvatures on the behaviour of the edge flame.

Figure 13(a) shows the joint PDF of $S_{e}^{*} / S_{L}$ and the prod- 

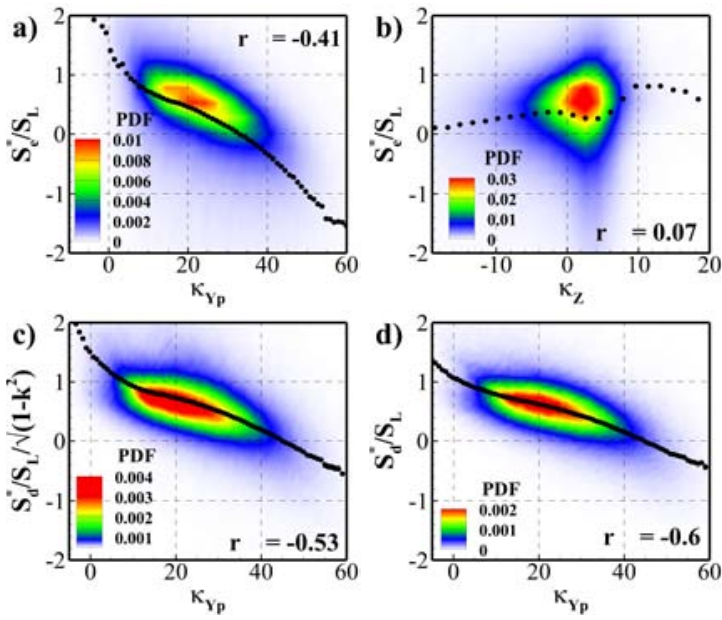

Figure 13: Contours of: the joint PDFs of $S_{e}^{*} / S_{L}$ with a) product mass-fraction iso-surface curvature $\kappa_{Y_{p}}$ and b) mixture-fraction isosurface curvature $\kappa_{Z} ; \mathrm{c}$ ) the joint PDF of the product mass-fraction displacement speed contribution to $S_{e}^{*} / S_{L}$ and $\kappa_{Y_{p}}$; d) the joint PDF of the product mass-fraction displacement speed and $\kappa_{Y_{p}}$.

uct mass-fraction curvature, $\kappa_{Y_{p}}$, whereas figure 13(b) shows the joint PDF of $S_{e}^{*} / S_{L}$ and the mixture-fraction curvature, $\kappa_{Z}$. It is first observed that the product mass-fraction curvatures are almost always positive, as observed in the laminar triple flames $[59,95]$ and unlike in fully premixed flames, where negative curvatures are observed frequently [30, 81 , 97, 98, 100]. This is because of the strong curvature of the product mass-fraction iso-surface in the plane $\boldsymbol{N}_{Z} \boldsymbol{T}_{2}$ - i.e. the plane containing the mixture-fraction variations and the direction of flame propagation. (Figure 2 can be consulted as a reminder of how these coordinates relate to the typical edgeflame structure.) In contrast to the product mass-fraction curvature, both positive and negative mixture-fraction curvatures are observed. Moving on to the correlations, it may be observed that the normalised edge-flame speed, $S_{e}^{*} / S_{L}$, is negatively correlated with the product mass-fraction curvature whereas it shows a weak positive correlation with the mixturefraction curvature. Because of the relatively weak and nonmonotonic trend shown for the effect of $\kappa_{Z}$ on $S_{e}$, a full analysis of this trend will not be presented. However, it is briefly noted that while the expected negative trend of $S_{Z}$ with $\kappa_{Z}$ was observed, it was counteracted by correlations of the orientation factor $-k / \sqrt{1-k^{2}}$ with curvature. The remaining discussion of $S_{e}$ trends will focus on the $S_{d}$ component.

To explain the physical reason for the $S_{e}$ curvaturecorrelations, individual components are now examined. Figures 13(c) presents the joint PDF of the terms involving in $S_{d}^{*}$ in equation 7 and the product mass-fraction curvature. As shown in figure 13(c), the term corresponding to $S_{d}^{*} / S_{L}$ exhibits a negative correlation with $\kappa_{Y_{p}}$. This is the main reason for the observed negative correlation for $S_{e}^{*} / S_{L}$ in figure 13(a).

Further drill-down is presented in figures 13(d) which shows the joint PDFs of $S_{d}^{*} / S_{L}$ with $\kappa_{Y_{p}}$. A clear negative
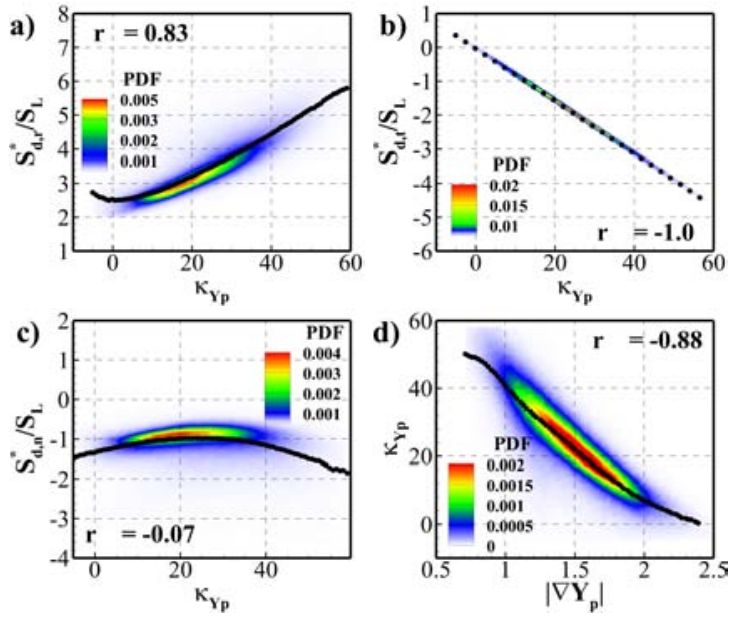

Figure 14: Contours of: the joint PDFs with product mass-fraction curvatures ( $\kappa_{Y_{p}}$ ) of a) $S_{d, r}^{*} / S_{L}$, b) $S_{d, t}^{*} / S_{L}$, c) $S_{d, n}^{*} / S_{L}$; and d) the joint PDF of $\kappa_{Y_{p}}$ and the magnitude of the product mass-fraction gradient.

correlation is observed. A negative correlation of the displacement speed and flame-front curvature was observed in previous studies of laminar edge flames [20], premixed turbulent flames [35, 38, 97], and turbulent edge flames [30]. This negative correlation was also observed in DNS study of methane premixed flame [97] where negative displacement speed reported in high positive flame front curvature. There is a good match for the qualitative trends with $\kappa_{Y_{p}}$ between $S_{d}^{*}$ and its component in $S_{e}^{*}$, suggesting that the orientation factor does not significantly change with curvature.

Understanding the behaviour observed in figures 13(d), requires the knowledge of the components of $S_{d}^{*}$ in response to product mass-fraction curvature. The joint PDF of the components of $S_{d}^{*} / S_{L}$ presented in equation 10 with the curvature of the product mass-fraction iso-surface are presented in figures 14(a)-(c). The reaction term is positive and increases with $\kappa_{Y_{p}}$ (figure 14(a)). As discussed earlier, the product mass-fraction reaction rate is approximately constant. Therefore, this strong positive correlation is linked to the response of curvature to the product mass-fraction gradient, which will be demonstrated below. The tangential term shown in figure 14(b) has the expected negative correlation as per equation 10 . The normal diffusion term presents a weak negative correlation with $\kappa_{Y_{p}}$. In contrast to the other two terms, it features a lower level of variations suggesting that it is not as important as the other terms in determining the overall trend.

To explain the trend for the reaction term, figure 14(d) shows the joint PDF of $\kappa_{Y_{p}}$ and the magnitude of the product mass-fraction gradient, $\left|\nabla Y_{p}\right|$. The curvature of the product mass-fraction iso-surface is negatively correlated with $\left|\nabla Y_{p}\right|$. As $S_{d, r}^{*} \approx 1 /\left|\nabla Y_{P}\right|$ this confirms that a $\left|\nabla Y_{p}\right|-\kappa_{Y_{p}}$ correlation is the reason for the positive $S_{d, r}^{*}-\kappa_{Y_{p}}$ correlation. Such a correlation is expected because the largest curvature of the product mass-fraction iso-surfaces is expected to be found in the $\boldsymbol{N}_{Z} \boldsymbol{T}_{2}$ plane (see figure 2 for a schematic). Compressive 

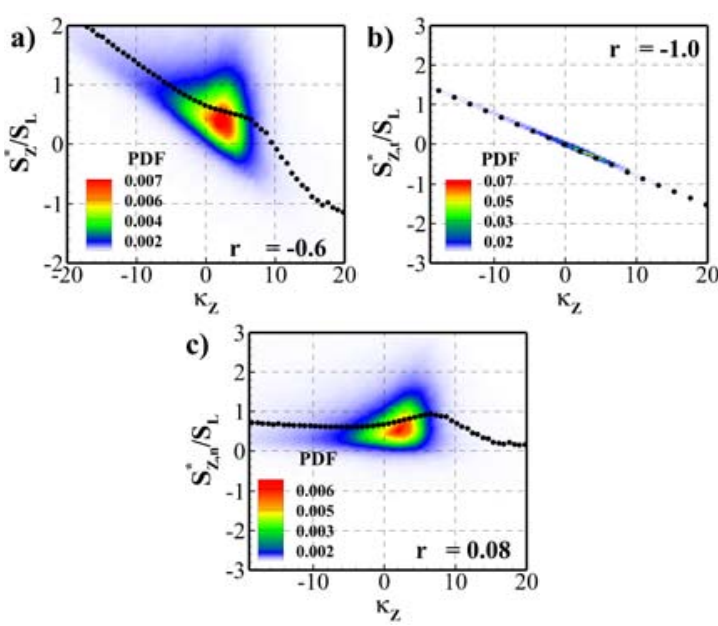

Figure 15: Contours of: the joint PDFs with mixture-fraction curvature of a) the mixture-fraction displacement speed b) tangential diffusion component; and c) normal diffusion component.

strain in the $N_{Z}$ direction therefore increases this curvature, while simultaneously decreasing the $\left|\nabla Y_{P}\right|$ since the $N_{Z}$ and $N_{Y_{p}}$ vectors are not generally aligned. Note that this physical picture is quite consistent with the earlier discussion of the negative correlation of scalar dissipation rate with $\kappa_{Y_{p}}$ that was discussed in relation to figure 10 (b) and the schematic in figure 11 .

The joint PDFs of $S_{Z}^{*} / S_{L}$ and its the components, equation 10 , and the mixture-fraction curvature, $\kappa_{Z}$, are presented in figure 15. A clear negative correlation between $S_{Z}^{*} / S_{L}$ and $\kappa_{Z}$ is observed (figure 15a). The tangential term shows the expected linear relationship dictated by equation 10 . However, within the region that contains most of the sample, the normal component shows only a weak positive correlation with the curvature leading us to conclude that the tangential term is the main reason for the observed negative correlation of $S_{Z}^{*}$ with $\kappa_{Z}$. The earlier discussion regarding the positive correlation between curvature and scalar dissipation rate in figure 12(d) explains the weak positive correlation between the normal diffusion term and $\kappa_{Z}$.

\subsubsection{Responses to strain}

Many previous studies of premixed flames and partiallypremixed edge flames $[30,81,97,98,100]$ have demonstrated strong effects of strain. These effects are therefore investigated in this section.

Figure 16(a) shows the joint PDF of $S_{d}^{*} / S_{L}$ and the tangential strain rate of the product mass-fraction iso-surfaces. For strain rates up to about $0.1 / t_{j}$, there is a positive correlation between $S_{d}^{*} / S_{L}$ and $a_{t, Y_{p}}$ while for higher strain rates, there is a negative correlation.

The above result can be explained considering the different effects of tangential strain according to the tangent orientation. Recall that the $\boldsymbol{T}_{1}$ direction is the mutual tangent vector between mixture-fraction and product mass-fraction iso- surfaces, while the $\boldsymbol{T}_{3}$ direction is the tangent to product massfraction iso-surfaces which is normal to $\boldsymbol{T}_{1}$. The alignment of $\boldsymbol{T}_{3}$ with $\boldsymbol{N}_{Z}$, which points towards the oxidiser side, tends to be good. Figure 16(b) shows the joint PDF $S_{d}^{*} / S_{L}$ with the strain rate $a_{T_{1}}$ in the $\boldsymbol{T}_{1}$ direction while figure 16(c) shows the PDF with strain rate $a_{T_{3}}$ in the $\boldsymbol{T}_{3}$ direction. While both of these strain rates show the same non-monotonic trend, the decreasing response of $S_{d}^{*}$ to increasing compressive strain (i.e. more negative) in the $\boldsymbol{T}_{3}$ direction is much stronger than the response in the $\boldsymbol{T}_{1}$ direction. In contrast, the decreasing response of $S_{d}^{*}$ to increasing extensive strain is much stronger in the $\boldsymbol{T}_{1}$ direction than it is in the $\boldsymbol{T}_{3}$ direction. In a statistically flat fully premixed flame, no statistical difference should be observed between strain rates in any two tangential directions. Moreover, it is well known that for unity Lewis number conditions, the $S_{d}^{*}$ has a decreasing response to strain $[81,98,100]$. This therefore suggests that the different response of the edge flame compared with a statistically flat premixed flame is due to curvatures. The largest curvature of the edge flame is generally found in the plane containing $\boldsymbol{T}_{3}$ and $\boldsymbol{T}_{2}$ - i.e. the curvature moving around the narrow flame edge from the rich to the lean side. A compressive strain in this direction causes the curvature to increase, which significantly decreases the flame speed, explaining the overall positive correlation to the strain in the $\boldsymbol{T}_{\mathbf{3}}$ direction. This picture is confirmed in figures 16(d) and (e), which plot the joint PDFs of product curvature and the strain rate in the $\boldsymbol{T}_{1}$ and $\boldsymbol{T}_{3}$ directions, respectively. As may be observed, the correlation for $a_{T_{3}}$ is strong and negative, and recalling figure 13(e), a larger product curvature leads to a lower flame displacement speed. In contrast, extensive strain in the direction of $\boldsymbol{T}_{1}$ does not affect the curvature, as the curvature is mainly found in the orthogonal $\boldsymbol{T}_{3}$ and $\boldsymbol{T}_{2}$ plane, as shown in figure $16(\mathrm{~d})$. Thus the strain tends to increase the product mass-fraction gradient, which decreases the reaction term in $S_{d}^{*}$ while leaving the normal diffusion term largely unaffected (c.f. figure 9(a) and the earlier discussion of the scalar dissipation effects in section 4.2.2). Considering the reaction term is decreased, and there is only a minimal effect on the curvature term, the overall $S_{d}^{*}$ decreases with increasing strain in the $\boldsymbol{T}_{1}$ direction. Summarising the non-monotonic trend for $S_{d}^{*}$ with tangential strain rate: the increasing trend from negative to weak positive strains is explained mainly by strain in the $\boldsymbol{T}_{3}$ direction, which causes curvature changes and thus lower $S_{d}$, while the decreasing trend for strongly positive curvatures is explained mainly by strain in the $\boldsymbol{T}_{1}$ direction, due to the scalar gradient increasing without large curvature changes. The observations for the strain in the $T_{3}$ direction are consistent with previous findings of Im and Chen [36] in a study of triple flame structure of a partially premixed hydrogen-air flame in which the negative displacement speed was observed to be induced by compressive strain and curvature. Extinction of the edge flame by extensive strain rate and increase of edge-flame curvature has also reported in the numerical study of laminar triple flames by Liao et al. [101] where single-step chemistry was applied.

To investigate strain effects on the mixture-fraction dis- 

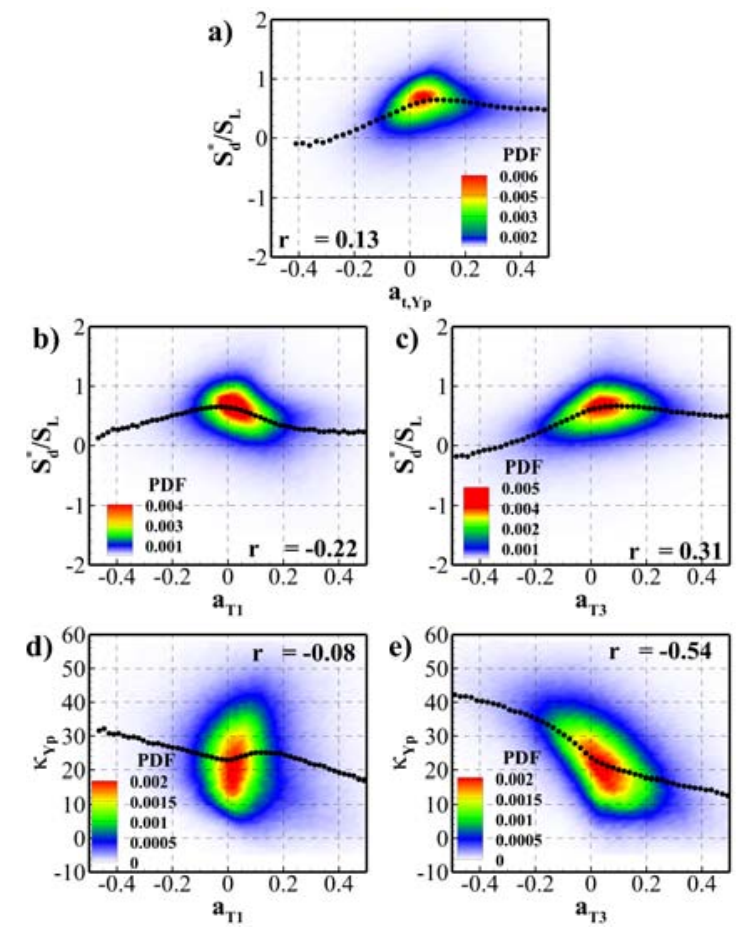

Figure 16: Contours of: the joint PDFs of a) the product mass-fraction displacement speed and the tangential strain rate of product massfraction iso-surfaces; the product mass-fraction displacement speed and the strain rate of product mass-fraction iso-surfaces in the b) $\boldsymbol{T}_{1}$ and c) $\boldsymbol{T}_{3}$ directions; the product mass-fraction curvature and the strain rate of product mass-fraction iso-surfaces in the d) $\boldsymbol{T}_{1}$ and e) $\boldsymbol{T}_{3}$ directions.
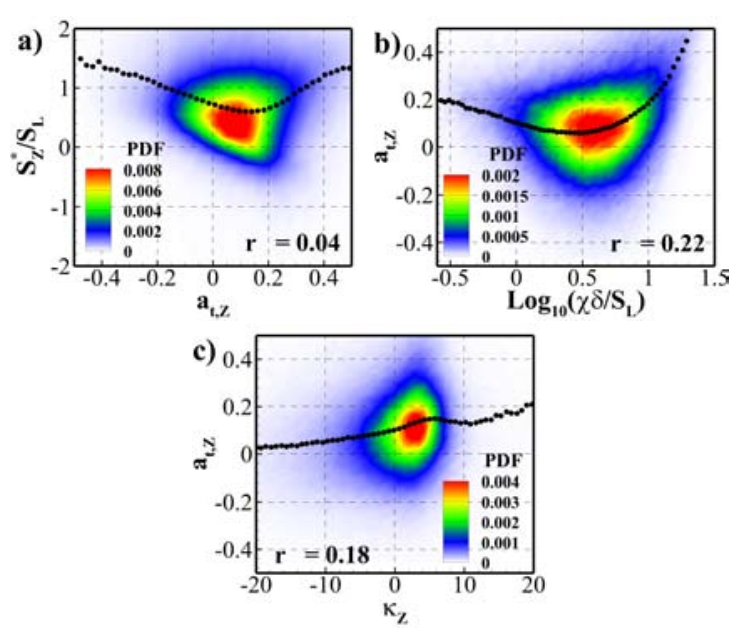

Figure 17: Contours of: the joint PDFs of a) $S_{Z}^{*} / S_{L}$ and the tangential strain rate of mixture-fraction iso-surfaces $a_{t, Z}$, b) $a_{t, Z}$ and the scalar dissipation rate, and c) $a_{t, Z}$ and the mixture-fraction curvature.

placement speed, figure 17 shows joint PDFs of: a) the normalised mixture-fraction displacement speed $S_{Z}^{*} / S_{L}$ and the tangential strain rate of mixture-fraction iso-surfaces $a_{t, Z}, \mathrm{~b}$ ) $a_{t, Z}$ and the scalar dissipation rate $\chi$, and c) $a_{t, Z}$ and the mixture-fraction curvature $\kappa_{Z}$. Figure 17(a), shows that like the product mass-fraction iso-surfaces, the relationship is nonmonotonic. The explanation however is more straightforward. The positive correlation on the high strain rate side is explained by a positive correlation between scalar dissipation rate and tangential strain rate at high strain rates which is shown in figure 17(b), recalling from the discussion of scalar dissipation effects in section 4.2.2 that $S_{Z}^{*} / S_{L}$ is positively correlated with $\chi$ (c.f. figure $8(\mathrm{e})$ ). On the compressive strain side, the effect is explained by a negative strain-curvature correlation, shown in figure $17(\mathrm{c})$. Since more negative curvatures increase the flame speed, this explains the negative correlation with strain.

Orientation effects are now considered. Figures 18(a) and (b) show the joint PDFs of the tangential strain rate of product mass-fraction (a) and mixture-fraction (b) iso-surfaces with the inner product of the normal vectors to these iso-surfaces, $k$. The PDFs have the expected shape. For small extensive strains, the alignment is weak and the correlation is not strong, while for larger extensive strains the normal vectors tend towards being aligned or anti-aligned, with the former possibility being much more likely.

\subsection{Spatially and temporally averaged edge-flame re- sponses}

In the last section, we discussed local correlations of the edge-flame speeds with various quantities, neglecting any spatial dependence. However, the spatial dependence is particularly significant given the flame undergoes on-average cyclical fluctuations of its location (recall figure 6). During different 

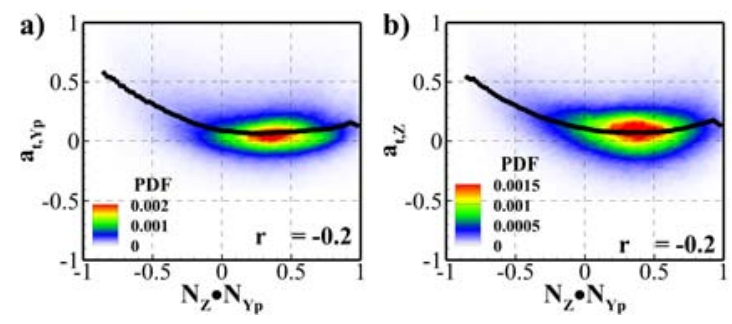

Figure 18: Contours of: the joint PDFs of the tangential strain rates of a) the product mass-fraction iso-surfaces and b) the mixture-fraction iso-surfaces with the inner product of the normal vectors to these isosurfaces $k$.
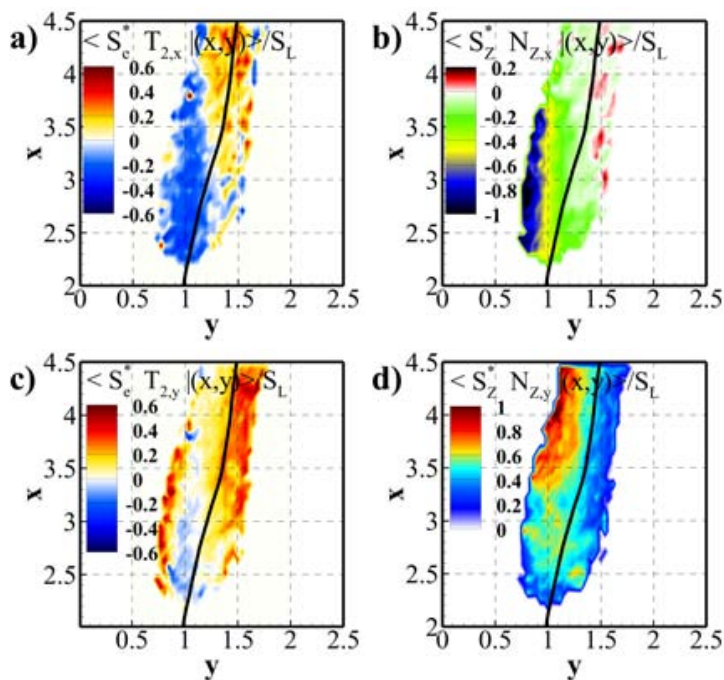

Figure 19: The following quantities, conditionally averaged on both streamwise and transverse locations of the instantaneous flame base: the streamwise component of a) $S_{e}^{*} / S_{L} T_{2}$ and b) $S_{Z}^{*} / S_{L} N_{Z}$; and the transverse component of c) $S_{e} T_{2}$ and d) $S_{Z}^{*} / S_{L} N_{Z}$. In all figures, the solid line is the temporally and spatially Favre-averaged mixture fraction equal to 0.07 .

phases of its cycle, it may experience different curvatures, dissipation rates and strain rates, and this implies it may undergo cyclic fluctuations of edge-flame speed and other variables as well. This is what is examined in this section.

Where possible in this section we will explain the observed spatial trends for dependent variables (such as the edge-flame speed) in terms of spatial variations of other variables (such as strain rate, curvature, etc.). However, a full understanding of the spatial variations also requires an overall picture of why different strains, curvatures, etc. are experienced at different phases of the cycle. This aspect is deferred to a later section which synthesises the overall picture of the edge-flame dynamics.

\subsubsection{Averaged edge-flame velocity components}

To begin, we first present the overall trends of the mean edge-flame velocity components before attempting to explain these by reference to other variables. Figures 19(a) and (b) present the streamwise component of $S_{e}^{*} / S_{L} T_{2}$ and $S_{Z}^{*} / S_{L} N_{Z}$, respectively, conditionally averaged at the instantaneous locations of the edge flames. (Henceforth, we will simply refer to this as "conditionally averaged" and the quantity as the "conditional mean".)

As can be seen both streamwise components are negative on the rich side at low lifted heights and contribute almost equally to the negative $V_{e, x}^{*} / S_{L}$ (figure 6(c)), indicating upstream propagation. (We remind the reader that in the discussion of these figures, when we refer to the rich or lean side, we mean the on-average rich or lean side.) At higher lifted heights, either on the rich or lean side, the streamwise component of $S_{e}^{*} / S_{L} \boldsymbol{T}_{2}$ is positive, whereas the streamwise component of $S_{Z}^{*} / S_{L} N_{Z}$ is negligible. Therefore, $S_{e}^{*} / S_{L}$ dominantly contributes to the positive $V_{e, x}^{*} / S_{L}$ at high lifted heights, figure $6 \mathrm{c}$.

Now turning to transverse propagation velocities (figures 19(c) and (d)), it is noted that the transverse component of $S_{e}^{*} / S_{L} \boldsymbol{T}_{2}$ is mostly positive except for a weakly negative central region in downstream areas. The transverse component of $S_{Z}^{*} / S_{L} N_{Z}$ is positive across all flame locations, and strongly positive on the rich side at high lifted heights. Once again, therefore, both transverse component of $S_{e}^{*} / S_{L} \boldsymbol{T}_{2}$ and $S_{Z}^{*} / S_{L} N_{Z}$ play a role to determine the net transverse edgeflame propagation in figure 6 . At large lifted heights, the contribution of $S_{Z} N_{Z, y}$ is dominant on the rich side, while $S_{e}^{*} T_{2, y}$ plays the leading role on the lean side.

To explain the above observations, both the effects of variations of edge-flame orientations and edge-flame speed need to be considered. We begin by considering the former. To do so, it is necessary to first understand the spatial trends for strain, since these significantly affect the orientations.

\subsubsection{Strain and flame orientation variations}

Figure 20(a) shows the conditionally averaged tangential strain rates of the mixture-fraction iso-surfaces, $a_{t, Z}$. It is readily observed that $a_{t, Z}$ is mostly positive, and strongest between around 10:30 though around 5 o'clock. Since mixture fraction is a non-reacting scalar, its normal is likely to align well with the most-compressive fluid dynamic strain rate [83, 102] such that the regions of high $a_{t, Z}$ are likely to correspond with regions of high fluid dynamic strain. It is proposed that high strains are observed in these regions due to passage of large eddies, which eject fluid outwards from the jet. The high strain period begins at the top of large eddy as it encounters the flame, and persists until the flame is entrained back into the jet core after the large eddy passes. (Further discussion will be given in the next section.)

The different strain rates experienced during the cycle are likely to affect the orientations of the edge flames and the relative normal to mixture-fraction and product mass-fraction isosurfaces. To demonstrate this point, figure 20(b) shows the conditional mean of the inner product $N_{Y_{p}}$ and $N_{Z}, k$. It may be observed that $k$ exhibits a bias towards alignment on the upper right side, and a bias towards non-alignment on the lower 
left, except on the far left. The trends are remarkably consistent with those for the tangential strain rate $a_{t, Z}$ as expected considering the greater ability of higher strain rates to align the normal vectors of different scalars.

The strain rates also affect the averaged orientations of the vectors. Figures 20 (c) and (d) show the conditionally averaged unit vector normal to the mixture-fraction iso-surfaces, $\boldsymbol{N}_{z}$, and the unit vector normal to the product mass-fraction iso-surfaces, $N_{Y_{p}}$, respectively. The unit normal vector of the mixture-fraction iso-surface is pointing towards the oxidiser at high lifted heights and turns somewhat towards clockwise at low lifted heights, indicating an on-average rotation of mixture-fraction iso-surfaces as they move through the clockwise rotating cycle of the flame edge around the mean stabilisation point. The vector $N_{Y_{p}}$ (and also $T_{2}$, not shown) goes through an even more significant rotation. Starting at large lifted heights on the lean side, around 1 o'clock, $N_{Y_{p}}$ points outwards towards the oxidiser, and is well aligned with the averaged $N_{Z}$. Moving downwards and inwards, through to 6 o'clock, $N_{Y_{p}}$ rotates clockwise and eventually points downwards towards unburned reactants (approximately in the 7 o'clock direction). From 6 o'clock through around 10:30, the flame maintains this orientation, until it suddenly changes from 10:30 through 12 o'clock to point outwards towards the oxidiser side. Explaining the trends for the on-average vector alignments will defer to the next section where they will be shown to be consistent with a proposed flame evolution resulting from the passage of large eddies.

The normalised flame index (NFI) [91, 103, 104], defined as

$$
\mathrm{NFI}=\frac{\nabla \mathrm{Y}_{\mathrm{F}} \bullet \nabla \mathrm{Y}_{\mathrm{O}}}{\left|\nabla \mathrm{Y}_{\mathrm{F}} \bullet \nabla \mathrm{Y}_{\mathrm{O}}\right|}
$$

can be used to distinguish between non-premixed and premixed burning. For premixed flames, the NFI $\rightarrow+1$ while for non-premixed flames the NFI $\rightarrow-1$. Figure 20(e) shows the conditionally averaged NFI. It may be observed that the NFI is positive on the lower left side (in rich, upstream regions), but negative on the upper rich side (across the whole lean side and also in rich, downstream regions). The mode of burning therefore is more premixed on the lower left than it is on the upper right, implying that the burning mode also undergoes cyclic fluctuations. These spatial fluctuations of NFI are proposed to be associated with the observed spatial fluctuations of strain rate which were earlier discussed. In the present situation of a flame in a mixing layer, positive NFI should only be expected as a result of the chemical reaction's effect on reactant gradients. In high strain regions, this effect is not fast enough and the alignment expected in the mixing layer dominates. As a result, in high strain regions, the conventional triple-flame structure cannot exist, causing the premixed branches to merge onto the diffusion flame and negative NFI to be observed.

\subsubsection{Displacement speed variations}

Variations of displacement speed also affect the averaged edge-flame propagation velocity components. The contribu-
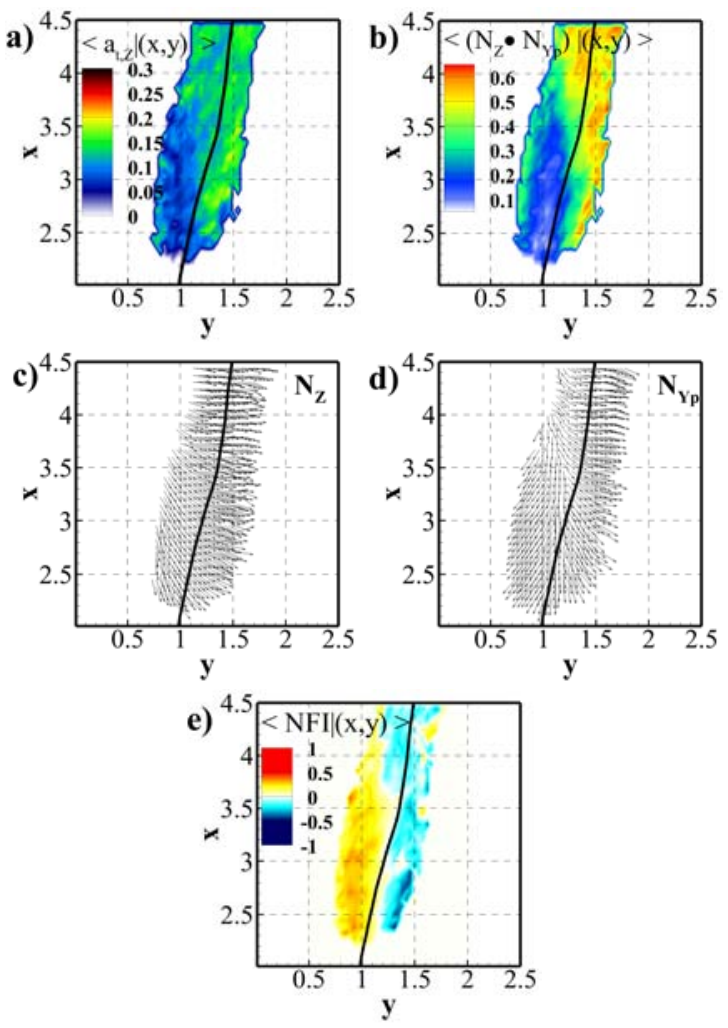

Figure 20: The conditionally averaged: (a) contours of tangential strain rates of the mixture-fraction iso-surfaces $a_{t, Z}$, (b) the inner product $k=N_{Z} \bullet N_{Y_{p}}$, (c) vectors normal to mixture-fraction iso-surfaces $N_{Z}$, (d) vectors tangential to product mass-fraction iso-surfaces $N_{Y_{p}}$, and (e) the normalised flame index (NFI). 

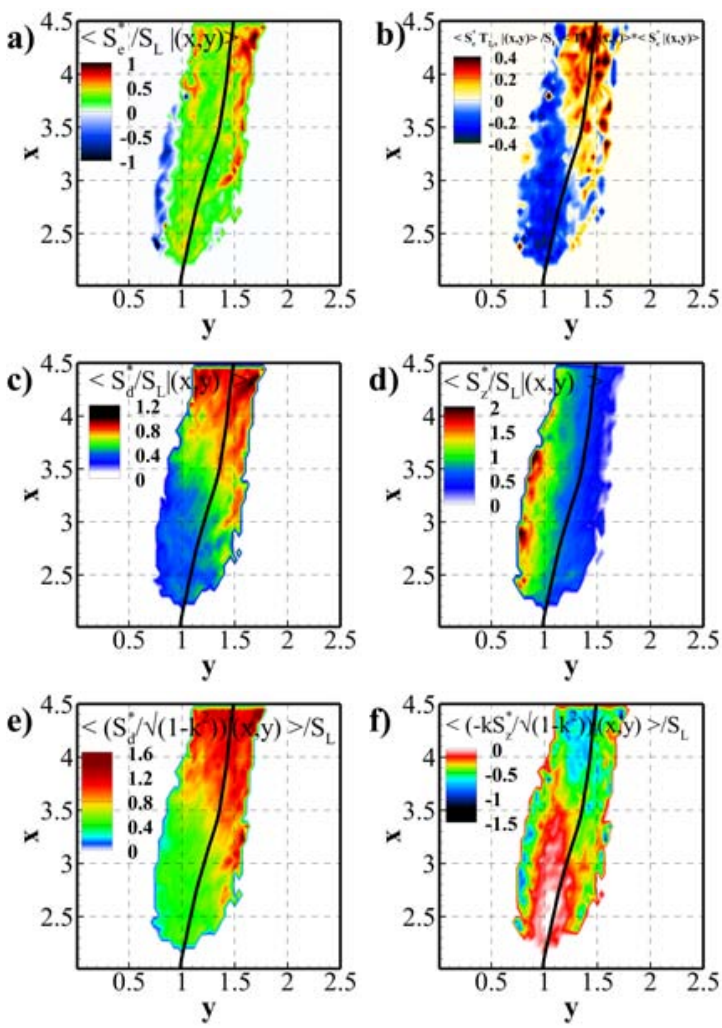

Figure 21: The following conditionally averaged quantities: a) the normalised edge-flame propagation speed in the $\boldsymbol{T}_{2}$ direction, $S_{e}^{*} / S_{L}$, b) the correlation of $S_{e}^{*} / S_{L}$ with the streamwise component of $\boldsymbol{T}_{2}$ c) the displacement speed of the product mass-fraction iso-surfaces, $S_{d}^{*} / S_{L}$, d) the displacement speed of the mixture-fraction iso-surfaces, $S_{Z}^{*} / S_{L}$, e) the contribution $S_{d}^{*} / S_{L}$ to $S_{e}^{*} / S_{L}$ f) the contribution of the $S_{Z}^{*} / S_{L}$ to $S_{e}^{*} / S_{L}$. The solid line is the temporally and spatially Favreaveraged mixture fraction equal to 0.07 . tion of the edge-flame speed $S_{e}^{*}$, which it is reminded represents the speed of flame propagation along mixture-fraction iso-surfaces towards reactants (see equation 7), is now investigated in more detail. Figure 21(a) presents the conditional average of $S_{e}^{*} / S_{L}$. It may be observed that $S_{e}^{*} / S_{L}$ is generally positive, except in a small region on the very rich side, but smaller than unity. It generally increases at locations further downstream. Combining this information with the averaged $N_{Y_{p}}$ vectors shown in figure 20 does not seem to explain the observed $<S_{e}^{*} / S_{L} \boldsymbol{T}_{2, x} \mid(x, y)>$ shown previously in figure 19, which was in the downstream direction on the upper right half of the plot. To explain this, figure 21(b) shows the correlation $<S_{e}^{*} / S_{L} \boldsymbol{T}_{2, x}>-<S_{e}^{*} / S_{L}><\boldsymbol{T}_{2, x}>$. Evidently, $S_{e}^{*}$ and $\boldsymbol{T}_{2, x}$ are highly correlated in the upper right region, explaining how the edge-flame propagation can on average be in the downstream direction in that region despite $S_{e}^{*}$ being on average positive.

To further investigate the causes of $S_{e}^{*}$ variations, figures 21(c) and (d) present the conditional average of $S_{d}^{*} / S_{L}$ and $S_{Z}^{*} / S_{L}$, respectively. The displacement speed of the product mass-fraction iso-surface, $S_{d}^{*} / S_{L}$, is generally positive. It is significantly less than unity in the upstream region and gradually increases to around unity as the edge flames move downstream as shown in figure 21(c). The reasons for this behaviour will be discussed later in detail shortly. The mixturefraction displacement speed, $S_{Z}^{*}$, (figure21(d)) is also positive with the highest values on the very rich side. Figures $21(\mathrm{e})$ and (f) present the conditionally averaged terms $S_{d}^{*} / S_{L} / \sqrt{1-k^{2}}$ and $-k S_{z}^{*} / S_{L} / \sqrt{1-k^{2}}$. As shown in equation 7 , these two terms contribute to the total edge speed, $S_{e}^{*}$. As can be seen comparing $S_{d}^{*} / S_{L} / \sqrt{1-k^{2}}$ to $S_{d}^{*}$ accounting for the orientation of the normal vectors (via the factor $1 / \sqrt{1-k^{2}}$ ) simply amplifies the $S_{d}^{*}$ contribution. In contrast, when the orientations are accounted for, $-k S_{z}^{*} / S_{L} / \sqrt{1-k^{2}}$ has a strong negative contribution, particularly in downstream regions and towards very rich and very lean regions in the upstream area. These trends on the alignment factors are consistent with the earlier discussed trends for $\mathrm{k}$, and therefore is also in turn explained by the effect of strain rates on the vector's alignments. Therefore, the negative $S_{e}^{*}$ on the rich side observed in figure 21(a), is principally the consequence of the negative contribution of $-k S_{z}^{*} / S_{L} / \sqrt{1-k^{2}}$. However, the regions of positive $S_{e}^{*}$ are due to the strong positive contribution from the term $S_{d}^{*} / S_{L} / \sqrt{1-k^{2}}$ slightly weakened by the negative contribution form the term $-k S_{z}^{*} / S_{L} / \sqrt{1-k^{2}}$.

The spatially averaged trends for $S_{d}^{*}$ are now discussed. It will be shown below that these are a result of both strain and curvature effects. Beginning with the strain, figure 22 shows the conditionally averaged tangential strain rate $a_{t, Y_{p}}$. Overall it shows an opposite trend from the mixture-fraction isosurfaces, $a_{t, Z}$, being larger on the bottom left (from 6 o'clock through around 10:30) than it is on the top right. ${ }^{2}$ Recalling the earlier discussion of strain rate effects in section 4.2.4,

\footnotetext{
${ }^{2}$ The fact that $a_{t, Y_{p}}$ shows the opposite trend from $a_{t, Z}$ on the lower
} 
$S_{d}^{*} / S_{L}$ is negatively correlated with $a_{t, Y_{p}}$, so on the rich side, this correlation explains the less than unity $S_{d}^{*} / S_{L}$. It does not, however, explain the low values on the lean side.

To explain the lean side behaviour, the different responses to strain in the tangent directions $\boldsymbol{T}_{1}$ and $\boldsymbol{T}_{3}$ need to be invoked. Figures 22(b) and (c) show the conditionally averaged strain rates in those directions, $a_{T_{1}}$ and $a_{T_{3}}$, respectively. Here, very different spatial patterns are observed. The strain rate in $\boldsymbol{T}_{3}$ direction is compressive, on-average in the region roughly between 3 and 5 o'clock. As noted in section 4.2.4, compressive strain in this direction causes the product mass-fraction curvatures to increase (c.f. figure 16(e)), which are also correlated well with high scalar dissipation rates (c.f. figure 10(b)). The high $\kappa_{Y_{p}}$ leads to a high tangential diffusion contribution, which ultimately leads to a lower $S_{d}^{*}$. To confirm this, figure 22(d) shows the conditionally averaged $\kappa_{Y_{p}}$ while figure 22(e) shows the dissipation rate $\chi$. As expected, both show high values between around 3 o'clock to around 6 o'clock, explaining the low $S_{d}^{*} / S_{L}$ in this region. It is also noted that the high dissipation rate values are consistent with persistent extensive tangential strain on mixture-fraction iso-surfaces on the lean side.

Finally, it is noted that $a_{T_{3}}$ is strongly extensive on the rich side, from 6 o'clock through to around 10:30. This leads to small product curvatures such that the curvature-effect is insignificant and the strain effect, which reduces $S_{d}^{*}$, is dominant. In the downstream regions, $a_{T_{3}}$ remains weakly extensive, reducing the curvature term, while the magnitude of $a_{T_{1}}$ decays such that $S_{d}^{*} / S_{L}$ can relax to unity.

Finally in this sub-section, the trends for $S_{Z}^{*}$ are examined. Figures 23(a) and (b) show the conditionally averaged tangential diffusion and normal diffusion contributions to $S_{Z}^{*} / S_{L}$. The tangential diffusion is negative on the lean side and strongly positive on the rich side. The normal diffusion term shows weaker spatial variations and remains on-average positive. There is some evidence of a downstream decay and a correspondence with regions of high scalar dissipation rate. To explain the tangential diffusion term, the conditional average of the mixture-fraction curvatures is shown in figure 23(c). As expected, the curvature on average is positive on the lean side and negative on the rich side. Also note that quite large negative values can be obtained on the rich side. These trends are consistent with large eddies pushing the flammable mixtures outwards, thus resulting in the positive curvatures on the lean side. As the eddy passes, the rapid entrainment towards the rich side causes large negative curvatures to develop at the bottom of the eddy. A schematic of this picture will be given later. The curvature effect therefore explains the large values

left is expected because in this region the normal vectors are not aligned (c.f. figure 20(b)). In the region between 10:30 and 6 o' clock, the values are perhaps slightly smaller than expected given the tendency towards alignment of the normals to product mass-fraction and mixture-fraction iso-surfaces, but the alignment is not perfect, with the inner product around 0.5 , which together with the lower peak values of the mixture-fraction strain, is probably sufficient to explain the low $a_{t, Y_{p}}$.
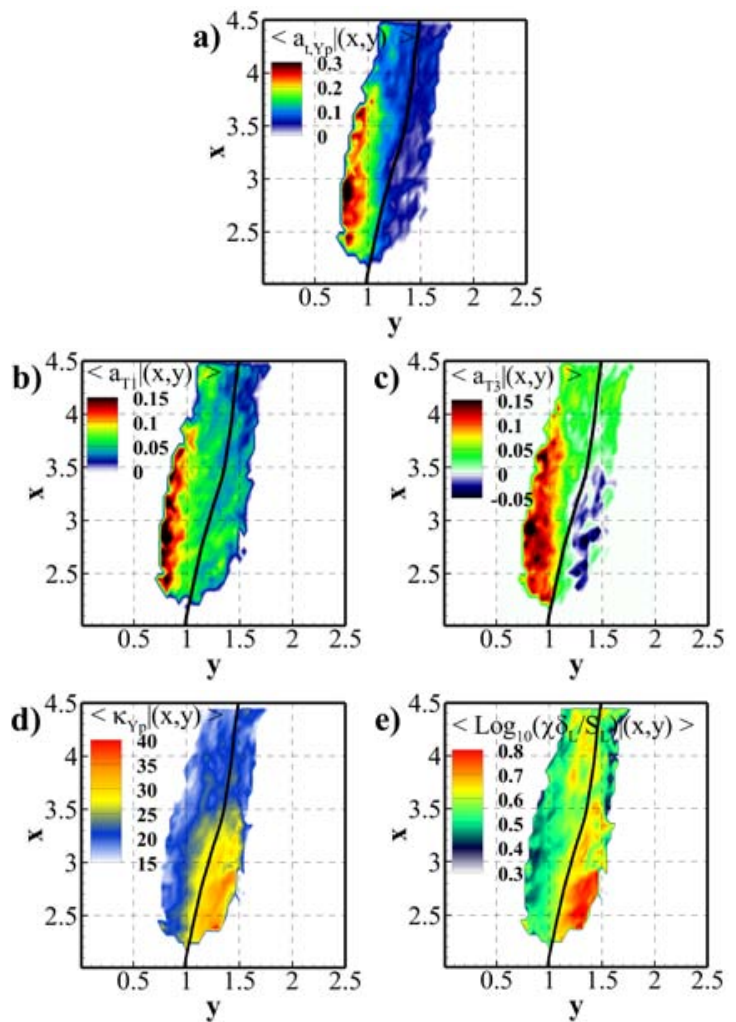

Figure 22: The conditional averages of: (a) product mass-fraction tangential strain rate $a_{t, Y p}$, (b) strain in the $\boldsymbol{T}_{1}$ direction, $a_{\boldsymbol{T}_{1}}$, (c) strain in the $\boldsymbol{T}_{3}$ direction, $a_{\boldsymbol{T}_{3}}$, (d) product mass-fraction curvature $\kappa_{Y_{p}}$, and e) scalar dissipation rate $\chi$. The solid line is the temporally and spatially Favre-averaged mixture fraction equal to 0.07 . 

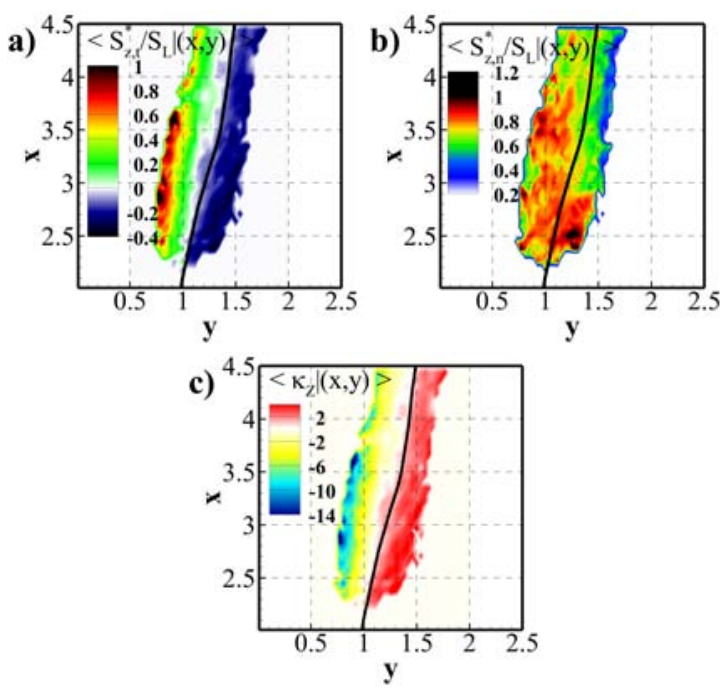

Figure 23: The conditional averages of: a) the tangential diffusion contribution to $S_{Z}^{*} / S_{L}$, (b) the normal diffusion contribution to $S_{Z}^{*} / S_{L}$, and mixture-fraction curvature. The solid line is the temporally and spatially Favre-averaged mixture fraction equal to 0.07.

attached by $S_{Z}^{*}$ on the rich side while the moderate values on the lean side are a result of a competition between the normal and tangential diffusion components.

\section{Discussion}

We begin the discussion by summarising the key points for the edge-flame velocity responses to local variables, as revealed by the PDFs presented in section 4.2. Next, we present an overall synthesis of the spatial picture.

Summarising the key points of edge velocity responses to the local scalar dissipation rate as:

- The edge-flame propagation velocity showed a negative correlation with the scalar dissipation rate consistent with previous studies [8, 13, 22, 23, 39, 90, 91]. For moderate dissipation rates, this was attributed mainly to the product mass-fraction $S_{d}^{*}$ component, while at higher dissipation rates, the normal vectors to product massfraction and mixture-fraction iso-surfaces tend to align causing the displacement speed of the mixture-fraction to decrease $S_{e}^{*}$.

- Analysing the components of $S_{d}^{*}$ revealed that the positive contribution of the reaction term with scalar dissipation rate is counteracted and overwhelmed by the negative contribution of the tangential term. The tangential term is driven by increases in product mass-fraction curvature while the reaction term is driven by increases in the product mass-fraction gradient. The increases in these controlling variables are in turn driven by compressive strain aligned approximately in the mixture-fraction normal direction, due to the typically poor alignment of the product normal with the mixture-fraction normal.
- Analysing the components of $S_{z}^{*}$ showed that the positive correlation of $S_{z}^{*}$ with the scalar dissipation rate is caused by its normal components. The normal component is controlled by mixing thickness which decreases with the scalar dissipation rate, as expected.

Turning to the edge-velocity responses to curvature:

- Edge-propagation velocity shows a negative correlation with product mass-fraction curvature which is mainly due to the negative correlation of $S_{d}^{*}$ with product massfraction curvature. The $S_{d}^{*}$ correlation is driven by the negative trend of the tangential diffusion component partially balanced by a competing trend of the reaction component, as previously discussed in relation to the scalar dissipation rate trend.

The summary of edge-velocity responses to the strain rates is as follows:

- The effect of strain on $S_{d}^{*}$ depended on its direction. The $S_{d}^{*}$ decreases as the strain rates compress in the surfaces in the both $\boldsymbol{T}_{1}$ and $\boldsymbol{T}_{3}$ directions. (The reader is reminded that $\boldsymbol{T}_{3}$ is a tangent to the product mass-fraction isosurfaces that is usually aligned with the mixture-fraction normal.) This is consistent with increases of curvature and scalar dissipation rate by tangential compression strains, revealing the trends with all three variables to be closely connected. In contrast to the $\boldsymbol{T}_{3}$ strain, more extensive strains in the $\boldsymbol{T}_{1}$ direction (the mutual tangent of product mass-fraction and mixture-fraction iso-surfaces) caused $S_{d}^{*}$ reductions, similar to the behaviour observed in statistically flat, near unity Lewis number, premixed flames [105].

- With increasing tangential strain rate of mixture-fraction iso-surfaces, the trend for $S_{z}^{*}$ was non-monotonic. For extensive strains a positive correlation between strain rate and dissipation leads to an overall positive correlation, while for compressive strains caused higher negative curvatures to arise, leading to an overall negative correlation.

The spatially-averaged picture is now synthesised taking into account cyclic fluctuations of the controlling variables whose effects were described above. It is proposed that the observations are consistent with the edge flames' interactions with large eddies. Referring to the schematic in figure 24, our previous article [1] demonstrated that an on-average elliptical clockwise motion of the edge flames occurs. It was proposed that this motion was due to the passage of large eddies, which was mostly consistent with the physical picture outlined by $\mathrm{Su}$ et al. [12]. The effects of the eddy on the edge flame's speed, orientation, curvature, and strain rate are now folded in to our earlier picture. Moving around the representative clock-face, the following picture is proposed:

\section{At 9 o'clock:}

The flame is between large eddies. The net motion is downstream and outwards. The flow is downstream and inwards. The downstream flow is counteracted by upstream propagation due mainly to the $S_{d}^{*}$ component of $S_{e}^{*}$. While $S_{e}^{*}$ is not 
a)

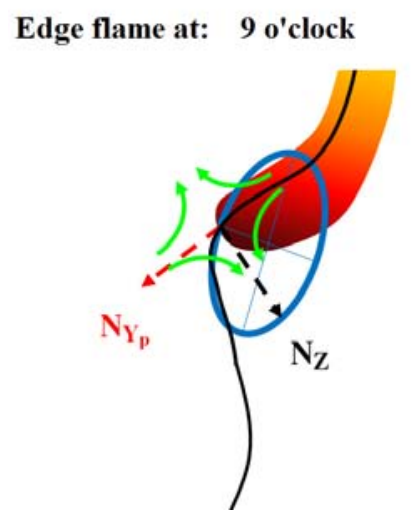

b)

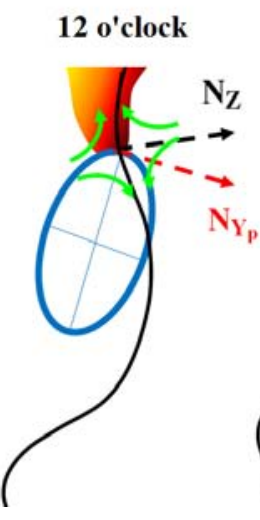

c)

3 o'clock d)

6 o'clock

Figure 24: A schematic to explain the edge flames response to the passage of a large eddy. The black line represents the mixture-fraction iso-surface. The red line represents the product mass-fraction iso-surface. The green curved arrows schematically represent the strain direction. The indicative directions of the normals to mixture-fraction and product mass-fraction iso-surfaces are shown as dashed arrows. The indicative clock-face is shown as the ellipse, with 9 o'clock, 12 o'clock, 3 o'clock and 6 o'clock marked.

large in this region, it is well correlated with the flame orientation being in the upstream direction. The inwards flow is counteracted by outwards flame propagation due mainly to high $S_{Z}^{*}$ as a result of high negative mixture-fraction curvatures ${ }^{3}$. In this region the normal vectors are not well aligned, and the flame tends towards having a more premixed character. There is extensive strain in the direction $\boldsymbol{T}_{3}$, which tends to be aligned with $N_{Z}$ causing low product curvature and low scalar dissipation rate. However, a large strain in the $\boldsymbol{T}_{1}$ direction (out-of-plane in the schematic) causes $S_{d}^{*}$ to be low.

\section{At 12 o'clock:}

The flame is at the top of a large eddy. The net motion is downstream and outwards. Although it has not been discussed in this article, extensive visualisations suggest that most edges that are moving downstream in this region are edges found on or about to form flame holes. These edges continue to move downstream until they are eventually annihilated. Edges that remain connected to the main leading flame however move outwards through this region. The streamwise flow is neutral and transverse flow is inwards but much smaller than in other regions. Flame propagation is outwards with contributions from both $S_{Z}^{*}$, and $S_{d}^{*}$. Mixture-fraction curvatures are low in this region. The tangential strain on mixture-fraction iso-surfaces is high as the large eddy begins to push the flame outwards. Between about 10:30 and 12 o'clock, the product mass-fraction iso-surfaces have rotated significantly such that now the normal vectors are relatively well aligned. In this phase of the cycle, the flame has a more non-premixed character than the other phases. There is moderate $\boldsymbol{T}_{3}$ and $\boldsymbol{T}_{1}$ strain,

\footnotetext{
${ }^{3}$ While it has not been discussed in the present article [1], our previous article shows that out-of-plane motion is also important in this region, allowing flames to escape regions of high inwards flow - the reader is referred to the earlier article for further discussion of this point.
}

and low product curvature which leads to a relatively large $S_{d}^{*}$ compared with the other regions.

\section{At 3 o'clock:}

The flame is now at the outer-most point of the large eddy. The net motion is upstream and inwards. The streamwise flow is upstream and the transverse flow is inwards. Flame propagation is outwards with contributions from both $S_{Z}^{*}$, and $S_{d}^{*}$, although the contribution of $S_{Z}^{*}$ is relatively weakened compared with other regions due to the high positive mixturefraction curvature, which reduces $S_{Z}^{*}$. The strain tangential to mixture-fraction iso-surfaces continues to be high and persistent tangential strain from 12 o'clock is increasing the scalar dissipation rate. The normals are still relatively well aligned, and relative to other regions, flame is less strongly premixed. However, from 3 o'clock through 6 o'clock, the alignment of the normals is decreasing and the $T_{3}$ strain starts to become large and compressive, resulting in high product curvatures appearing through to 6 o'clock, and this decreases $S_{d}^{*}$.

\section{At 6 o'clock:}

Finally, at 6 o'clock, the flame is at the bottom of the large eddy. The net motion is inwards. The streamwise flow is small and downstream, but the transverse flow is strongly entraining, creating a hook-like structure. Flame propagation is upstream and outwards, but relatively small in this region. The product mass-fraction normal has now rotated so that the normal vectors are not well aligned, and the NFI is neutral. Tangential strain on mixture-fraction iso-surfaces is now small. Product curvature and scalar dissipation are still high and the $\boldsymbol{T}_{1}$-direction strain is beginning to increase, leading to low $S_{d}^{*}$ persisting. The strain in the $\boldsymbol{T}_{3}$ direction has switched to extensive, so that the product curvature decreases from this point through to 9 o'clock. 


\section{Conclusions}

Edge flames have been studied in terms of their propagation speeds, the orientations of the normal vectors to mixturefraction and product mass-fraction iso-surfaces, the curvatures of those iso-surfaces, strain rates in various directions, and gradients of product mass fraction and mixture fraction.

The key findings are as follows.

- Significant instantaneous fluctuations of these quantities were observed, as well as on-average variations depending on where the edge flame was located.

- The net flame relative propagation speeds are affected instantaneously and on-average by contributions from the displacement speeds of the product mass-fraction and mixture-fraction iso-surfaces as well as the orientations of the normal vectors. None of these influences can be ignored to fully understand the edge-flame behaviour.

- The displacement speed of the product mass-fraction is strongly reduced by the product curvature, which is enhanced when a compressive strain is oriented in the tangent direction $\boldsymbol{T}_{3}$ (pointing across the edge-flame, usually in the direction of the mixture-fraction normal). As compressive strain in this direction also usually leads to mixture-fraction gradients increasing, a negative correlation with the scalar dissipation rate is also observed. Large extensive strain in the tangent direction $\boldsymbol{T}_{1}$ (the mutual tangent of product mass-fraction and mixturefraction iso-surfaces) also causes $S_{d}^{*}$ to diminish.

- Variations of the mixture-fraction displacement speed are affected most strongly by mixture-fraction curvature, but normal diffusion plays a role to offset the displacement speed to positive values (towards the oxidiser side) on average.

- As the flame moves around the on-average stabilisation point (outwards, then downstream, then inwards, and upstream again), it undergoes on-average cyclic variations of the various flame speeds involved, the orientations of the normal vectors, gradients, and curvatures. It is proposed that these on-average variations are connected with the passage of large eddies in a way that was described schematically in figure 24 . The overall picture is consistent with, but further elaborates, earlier proposals of roles played by large eddies by $\mathrm{Su}$ et al. [12] and our earlier article Karami et al. [1], which in turn drew on several previous works including notably Refs. $[49,87,106]$.

In future work, our priorities include determining whether and how this picture depends on: lifted height, Lewis number, dilution, and configuration (e.g. slot versus round jet).

Finally, it is noted that future detailed chemistry and transport DNS should be conducted to confirm and refine the present work. While we expect that qualitative trends for responses of the edge-flame speeds to imposed curvature and strain would be similar between detailed and simple models, quantitatively we would expect some differences.

\section{Acknowledgement}

This work was supported by the Australian Research Council. The research benefited from computational resources provided through the National Computational Merit Allocation Scheme, supported by the Australian Government. The computational facilities supporting this project included the Australian NCI National Facility, the partner share of the NCI facility provided by Intersect Australia Pty Ltd., the Peak Computing Facility of the Victorian Life Sciences Computation Initiative (VLSCI), iVEC (Western Australia), and the UNSW Faculty of Engineering. This research was sponsored by the US Department of Energy, Office of Basic Energy Sciences, Division of Chemical Sciences, Geosciences, and Biosciences. Sandia National Laboratories is a multi-program laboratory operated by Sandia Corporation, a Lockheed Martin Company, for the US Department of Energy under Contract DE-AC04-94-AL85000.

\section{References}

[1] S. Karami, E.R. Hawkes, M. Talei, J.H. Chen, J. Fluid Mech. 777 (2015) 633-689.

[2] S.H. Stårner, R.W. Bilger, J.H. Frank, D.F. Marran, M.B. Long, Combust. Flame 107 (1996) 307 - 313.

[3] L. Muñiz, M.G. Mungal, Combust. Flame 111 (1997) 16-31.

[4] K.A. Watson, K.M. Lyons, J.M. Donbar, C.D. Carter, Combust. Sci. Technol. 175 (2003) 649-664.

[5] K.A. Watson, K.M. Lyons, J.M. Donbar, C.D. Carter, Combust. Flame 123 (2000) 252 - 265.

[6] E.F. Hasselbrink Jr, M.G. Mungal, Symp. (Int.) Combust. 27 (1998) 867-873.

[7] C.M. Arndt, R. Schiel, J.D. Gounder, W. Meier, M. Aigner, Proc. Combust. Inst. 34 (2013) 1483 - 1490

[8] J. Boulanger, L. Vervisch, J. Reveillon, S. Ghosal, Combust. Flame 134 (2003) 355-368.

[9] L. Vanquickenborne, A. van Tiggelen, Combust. Flame 10 (1966) $59-69$.

[10] G.T. Kalghatgi, Combust. Sci. Technol. 41 (1984) 17-29.

[11] R.W. Schefer, M. Namazian, J. Kelly, Combust. Flame 99 (1994) $75-86$

[12] L.K. Su, O.S. Sun, M.G. Mungal, Combust. Flame 144 (2006) 494-512.

[13] J. Buckmaster, Combust. Sci. Technol. 115 (1996) 41-68.

[14] N. Peters, F.A. Williams, AIAA Journal 21 (1983) 423-429.

[15] C.J. Lawn, Prog. Energy Combust. Sci. 35 (2009) 1-30.

[16] W.M. Pitts, Combust. Flame 76 (1989) 197-212.

[17] K.M. Lyons, K.A. Watson, C.D. Carter, J.M. Donbar, Combust. Sci. Technol. 179 (2007) 1029-1037.

[18] H. Phillips, Symp. (Int.) Combust. 10 (1965) 1277-1283.

[19] P.N. Kioni, B. Rogg, K.N.C. Bray, A. Liñán, Combust. Flame 95 (1993) 276-290.

[20] Y.S. Ko, S.H. Chung, Combust. Flame 118 (1999) 151-163.

[21] V.S. Santoro, A. Liñán, A. Gomez, Proc. Combust. Inst. 28 (2000) 2039-2046.

[22] S. Ghosal, L. Vervisch, J. Fluid Mech. 415 (2000) 227-260.

[23] J. Buckmaster, Prog. Energy Combust. Sci. 28 (2002) 435-475.

[24] V. Favier, L. Vervisch, Combust. Flame 125 (2001) 788-803.

[25] V. Favier, L. Vervisch, Proc. Combust. Inst. 27 (1998) 12391245 .

[26] S.H. Chung, Proc. Combust. Inst. 31 (2007) $877-892$.

[27] H.G. Im, J.H. Chen, Combust. Flame 126 (2001) 1384-1392. 
[28] C. Jiménez, B. Cuenot, Proc. Combust. Inst. 31 (2007) 1649 1656.

[29] N. Chakraborty, E. Mastorakos, Flow Turbul. Combust. 80 (2008) 155-186.

[30] N. Chakraborty, E. Mastorakos, Phys. Fluids 18 (2006) 105103.

[31] H. Hesse, N. Chakraborty, E. Mastorakos, Proc. Combust. Inst. 32 (2009) 1399-1407.

[32] N. Chakraborty, H. Hesse, E. Mastorakos, Combust. Sci. Technol. 182 (2010) 1747-1781.

[33] T. Echekki, J.H. Chen, Combust. Flame 134 (2003) 169 - 191.

[34] T. Echekki, J.H. Chen, Combust. Flame 114 (1998) 231 - 245.

[35] T. Echekki, J.H. Chen, Combust. Flame 106 (1996) 184-202.

[36] H.G. Im, J.H. Chen, Combust. Flame 119 (1999) 436-454.

[37] J.H. Chen, E.R. Hawkes, R. Sankaran, S.D. Mason, H.G. Im, Combust. Flame 145 (2006) 128-144.

[38] J.H. Chen, H.G. Im, Symp. (Int.) Combust. 27 (1998) 819-826.

[39] C. Pantano, J. Fluid Mech. 514 (2004) 231-270.

[40] G.R. Ruetsch, L. Vervisch, A. Liñán, Phys. Fluids 7 (1995) 1447-1454.

[41] I.G. Boxx, W. Meier, C.D. Carter, AIAA SciTech, 52nd Aerospace Sciences Meeting (2014) AIAA 2014-0316.

[42] J. Oh, Y. Yoon, Int. J. Hydrogen Energ. 35 (2010) 1056910579.

[43] I. Boxx, C. Heeger, R. Gordon, B. Böhm, M. Aigner, A. Dreizler, W. Meier, Proc. Combust. Inst. 32 (2009) 905-912.

[44] A. Cessou, C. Maurey, D. Stepowski, Combust. Flame 137 (2004) 458-477.

[45] M.S. Mansour, Exp. Therm. Fluid Sci. 28 (2004) 771-779.

[46] A. Upatnieks, J.F. Driscoll, S.L. Ceccio, Proc. Combust. Inst. 29 (2002) 1897-1903.

[47] K.A. Watson, K.M. Lyons, J.M. Donbar, C.D. Carter, Proc. Combust. Inst. 29 (2002) 1905-1912.

[48] D. Demare, F. Baillot, Phys. Fluids 13 (2001) 2662-2670.

[49] J.B. Kelman, A.J. Eltobaji, A.R. Masri, Combust. Sci. Technol. 135 (1998) 117-134.

[50] M.M. Tacke, D. Geyer, E.P. Hassel, J. Janicka, Symp. (Int.) Combust. 27 (1998) 1157-1165.

[51] K.A. Watson, K.M. Lyons, J.M. Donbar, C.D. Carter, Combust. Flame 119 (1999) 199-202.

[52] R.W. Schefer, P.J. Goix, Combust. Flame 112 (1998) 559 574.

[53] R.W. Schefer, Combust. Sci. Technol. 126 (1997) 255-279.

[54] R.W. Schefer, Combust. Sci. Technol. 125 (1997) 371-394.

[55] C.M. Müller, H. Breitbach, N. Peters, Symp. (Int.) Combust. 25 (1994) 1099 - 1106.

[56] R.W. Schefer, M. Namazian, E.E.J. Filtopoulos, J. Kelly, Symp. (Int.) Combust. 25 (1994) 1223 - 1231.

[57] A. Upatnieks, J.F. Driscoll, C.C. Rasmussen, S.L. Ceccio, Combust. Flame 138 (2004) 259 - 272.

[58] J. Hult, U. Meier, W. Meier, A. Harvey, C.F. Kaminski, Proc. Combust. Inst. 30 (2005) 701-709.

[59] I.A. Mulla, S.R. Chakravarthy, Combust. Flame 160 (2013) 1345-1356.

[60] R.L. Gordon, I. Boxx, C. Carter, A. Dreizler, W. Meier, Flow Turbul. Combust. 88 (2012) 503-527.

[61] C. Heeger, B. Böhm, S.F. Ahmed, R. Gordon, I. Boxx, W. Meier, A. Dreizler, E. Mastorakos, Proc. Combust. Inst. 32 (2009) 2957-2964.

[62] S. Noda, H. Mori, Y. Hongo, M. Nishioka, JSME Int. J. B-Fluid T 48 (2005) 75-82.

[63] P.S. Kothnur, M.S. Tsurikov, N.T. Clemens, J.M. Donbar, C.D. Carter, Proc. Combust. Inst. 29 (2002) 1921-1927.

[64] B. Coriton, A.M. Steinberg, J.H. Frank, Exp. Fluids 55 (2014) 1743-1763.
[65] P. Domingo, L. Vervisch, Symp. (Int.) Combust. 26 (1996) 233-240.

[66] E. Mastorakos, T.A. Baritaud, T.J. Poinsot, Combust. Flame 109 (1997) 198-223.

[67] E.R. Hawkes, R. Sankaran, J.H. Chen, 16th Australasian Fluid Mechanics Conference (2007) 1271-1274

[68] C.S. Yoo, R. Sankaran, J.H. Chen, J. Fluid Mech. 640 (2009) 453-481.

[69] C.S. Yoo, E.S. Richardson, R. Sankaran, J.H. Chen, Proc. Combust. Inst. 33 (2011) 1619-1627.

[70] K.A. Watson, K.M. Lyons, J.M. Donbar, C.D. Carter, Combust. Flame 117 (1999) 257 - 271.

[71] S. Karami, E.R. Hawkes, M. Talei, J.H. Chen, Proceedings of the 2014 Summer Program, Center for Turbulence Research (Stanford, CA) (2015) 137-146.

[72] S. Karami, E.R. Hawkes, M. Talei, 18th Australasian Fluid Mechanics Conference (2012) 149.

[73] J.H. Chen, A. Choudhary, B. De Supinski, M. Devries, E.R. Hawkes, S. Klasky, W.K. Liao, K.L. Ma, J. Mellor-Crummey, N. Podhorszki, R. Sankaran, S. Shende, C.S. Yoo, Comput. Sci. Disc. 2 (2009).

[74] D. Garrido-López, S. Sarkar, Proc. Combust. Inst. 30 (2005) $621-628$.

[75] C.A. Kennedy, M.H. Carpenter, Appl. Numer. Math. 14 (1994) $397-433$.

[76] M. Tanahashi, M. Fujimura, T. Miyauchi, Proc. Combust. Inst. 28 (2000) $529-535$.

[77] E.R. Hawkes, J.H. Chen, Combust. Flame 138 (2004) 242258.

[78] E.R. Hawkes, J.H. Chen, Combust. Flame 144 (2006) 112125.

[79] E.R. Hawkes, R. Sankaran, J.C. Sutherland, J.H. Chen, Proc. Combust. Inst. 31 (2007) 1633-1640.

[80] R. Sankaran, E.R. Hawkes, J.H. Chen, T. Lu, C.K. Law, J. Phys: Conf Ser. 46 (2006) 38.

[81] N. Chakraborty, S. Cant, Combust. Flame 137 (2004) 129-147.

[82] E.R. Hawkes, R. Sankaran, J.H. Chen, Proceedings of the Australian Combustion Symposium (2007) 46-49.

[83] C.H. Gibson, W.T. Ashurst, A.R. Kerstein, J. Fluid Mech. 194 (1988) 261-293.

[84] S.B. Pope, Int. J. Eng. Sci. 26 (1988) 445-469.

[85] S.M. Candel, T.J. Poinsot, Combust. Sci. Technol. 70 (1990) $1-15$.

[86] C. Maurey, A. Cessou, B. Lecordier, D. Stepowski, Proc. Combust. Inst. 28 (2000) 545-551.

[87] R.C. Miake-Lye, J.A. Hammer, Proc. Combust. Inst. 22 (1989) 817-824.

[88] F. Baillot, D. Demare, Combust. Sci. Technol. 174 (2002) 7398.

[89] M. Namazian, R.W. Schefer, J. Kelly, Combust. Flame 74 (1988) 147-160.

[90] D. Veynante, A. Trouvé, K.N.C. Bray, T. Mantel, J. Fluid Mech. 332 (1997) 263-293.

[91] P. Domingo, L. Vervisch, J. Réveillon, Combust. Flame 140 (2005) $172-195$.

[92] J. Daou, A. Liñán, Combust. Flame 118 (1999) 479-488.

[93] E.R. Hawkes, R. Sankaran, J.H. Chen, in: 5th Joint US Combustion Meeting of the Combustion Institute (2007) B05.

[94] A. Juneja, S.B. Pope, Phys. Fluids 8 (1996) 2161-2184.

[95] X. Qin, C.W. Choi, A. Mukhopadhyay, I.K. Puri, S.K. Aggarwal, V.R. Katta, Combust. Theor. Model. 8 (2004) 293-314.

[96] A. Trouvé, T. Poinsot, J. Fluid Mech. 278 (1994) 1-31.

[97] I.R. Gran, T. Echekki, J.H. Chen, Symp. (Int.) Combust. 26 (1996) 323 - 329.

[98] E.R. Hawkes, J.H. Chen, Proc. Combust. Inst. 30 (2005) 647- 
655

[99] N. Chakraborty, S. Cant, Phys. Fluids 19 (2007) 105101.

[100] N. Chakraborty, E.R. Hawkes, J.H. Chen, R.S. Cant, Combust. Flame 154 (2008) 259-280.

[101] K.P. Liao, M. Matalon, C. Pantano, Proc. Combust. Inst. 35 (2015) 1015-1021.

[102] W.T. Ashurst, A.R. Kerstein, R.M. Kerr, C.H. Gibson, Phys. Fluids 30 (1987) 2343-2353.

[103] H. Yamashita, M. Shimada, T. Takeno, Symp. (Int.) Combust. 26 (1996) $27-34$.

[104] K. Bray, P. Domingo, L. Vervisch, Combust. Flame 141 (2005) $431-437$

[105] K.W. Jenkins, M. Klein, N. Chakraborty, R.S. Cant, Combust. Flame 145 (2006) 415-434.

[106] J.E. Broadwell, W.J. Dahm, M.G. Mungal, Symp. (Int.) Combust. 20 (1985) 303-310. 